\title{
HOW TO STATE NECESSARY OPTIMALITY CONDITIONS FOR CONTROL PROBLEMS WITH DEVIATING ARGUMENTS?
}

\author{
LASSANA SAMASSI $^{1}$ AND RABAH TAHRAOUI ${ }^{1}$
}

Abstract. The aim of this paper is to give a general idea to state optimality conditions of control problems in the following form:

$$
\inf _{(u, v) \in \mathcal{U}_{a d}} \int_{0}^{1} f\left(t, u\left(\theta_{v}(t)\right), u^{\prime}(t), v(t)\right) \mathrm{d} t
$$

where $\mathcal{U}_{a d}$ is a set of admissible controls and $\theta_{v}$ is the solution of the following equation:

$$
\begin{cases}\frac{\mathrm{d} \theta(t)}{\mathrm{d} t}=g(t, \theta(t), v(t)), & t \in[0,1] \\ \theta(0)=\theta_{0}, \quad \theta(t) \in[0,1] & \forall t .\end{cases}
$$

The results are nonlocal and new.

Mathematics Subject Classification. 49J15, 49J22, 49J25, 49J45, 49K15, 49K25, 49K22, 34K35, 47E05, 91B26, 91B28, 93C15.

Received June 13, 2006.

Published online November 21, 2007.

\section{INTRODUCTION}

A large class of physical problems deals with finding extrema of functional $J$ defined on some functional subspace $W$ of classical one like Sobolev space and which has the following form:

$$
J(u)=\int_{0}^{1} f\left(x, u(\theta(x)), u^{\prime}(\varphi(x))\right) \mathrm{d} x,
$$

where $\theta($.$) and \varphi($.$) are functions which satisfy, for instance, \theta([0,1])=[0,1], \varphi([0,1])=[0,1]$. We call $\theta($. and $\varphi($.$) deviations or deviating functions. The goal is to find a function u:[0,1] \longrightarrow \mathbb{R}$ belonging to $W$ and minimizing $J($.$) . We denote this problem by:$

$$
\inf [J(u) / u \in W] .
$$

Keywords and phrases. Functionals with deviating arguments, optimal control, Euler-Lagrange equation, financial market.

1 Ceremade, Université Paris IX-Dauphine, France; samassi@ceremade.dauphine.fr; tahraoui@ceremade.dauphine.fr 
Such problems have been studied by many people: for instance we can see $[5,9,11,17]$ and references of these works. If a solution $\bar{u}$ of (4) exists, then we can ask the following natural question: how to establish EulerLagrange equation of (4)? We use this terminology by analogy with the classical one.

From $[5,9,11]$, it is easy to see that necessary optimality conditions for (4) cannot be obtained in an easy way as the classical case. Let us denote by $(x, \eta, \xi)$ the respectives arguments of $f$. In [5] under suitable assumptions, the authors state the following functional differential equation:

$$
\left[\int_{0}^{1} \sigma_{\theta}(x, t) \frac{\partial f}{\partial \eta}\left(x, \bar{u}(\theta(x)), \bar{u}^{\prime}(\varphi(x))\right) \mathrm{d} x\right]-\frac{\mathrm{d}}{\mathrm{d} t}\left[\int_{0}^{1} \sigma_{\varphi}(x, t) \frac{\partial f}{\partial \xi}\left(x, \bar{u}(\theta(x)), \bar{u}^{\prime}(\varphi(x))\right) \mathrm{d} x\right]=0,
$$

where $W$ is a functional space of absolutely continuous functions with the derivatives in a Lebesgue space, $\bar{u}$ a solution of (4) and the functions $\sigma_{\theta}(.,$.$) and \sigma_{\varphi}(.,$.$) are defined by:$

$$
\sigma_{\theta}(x, t)= \begin{cases}1 & \text { if } \theta(x) \in[t, 1] \\ 0 & \text { if not. }\end{cases}
$$

Similar notation is used for $\varphi($.$) , with \varphi$ satisfying the following condition: $(\star)$ for any measurable subset $e$ of $[0,1]$ such that $|e|=0$ we have $\left|\varphi^{-1}(e)\right|=0$, where $|e|$ stands for Lebesgue measure of $e$. Let us point out that in (5) all the arguments $\bar{u}(\theta()),. \bar{u}^{\prime}(\varphi()$.$) are in the average form: (5) is not local - it is a functional$ differential equation - and seems to be far away from the classical Euler equation. In our approach we propose a general idea to state necessary conditions of optimality for a large class of problems. Our method applied to problem (4) gives the following nonlocal ordinary differential equation:

$$
\frac{\partial}{\partial \eta}\left\langle\nu_{t}^{\theta}, f\left(., \bar{u}(t), \bar{u}^{\prime}(\varphi(.))\right)\right\rangle+\sum_{i \in I} \delta_{t_{i}} \int_{\left\{\theta=t_{i}\right\}} \frac{\partial f}{\partial \eta}\left(s, \bar{u}(\theta(s)), \bar{u}^{\prime}(\varphi(s))\right) \mathrm{d} s-\frac{\mathrm{d}}{\mathrm{d} t}\left[\frac{\partial}{\partial \xi}\left\langle\mu_{t}^{\varphi}, f\left(., \bar{u}(\theta(.)), \bar{u}^{\prime}(t)\right)\right\rangle\right]=0,
$$

in the distribution space $\mathcal{D}_{t}^{\prime}(0,1)$. The measures $\nu_{t}^{\theta}$ and $\mu_{t}^{\varphi}$ stand for two counting measures defined from, respectively, the deviations $\theta($.$) and \varphi($.$) and \delta_{t_{i}}$ stands for Dirac measure at $t_{i}$. I is a subset of $\mathbb{N}$, possibly empty and the real numbers $t_{i}$ verify:

$$
\left|\left\{s \in[0,1] / \theta(s)=t_{i}\right\}\right|>0 \quad \forall i \in I .
$$

Let us point out that in (6) there are some arguments which are not in average form: $\bar{u}($.$) and \bar{u}^{\prime}($.$) . The singular$ character expressed by the Dirac measure is absent from the previous works. The method used in [5] is based on a formal abstract framework given in [2] - cf. also [3]. Our method uses a completely different argument: a desintegration result which is presented in general framework in [1]. For any $\xi=\left(\xi_{1}, \ldots, \xi_{n}\right) \in \mathbb{R}^{N}, N \geq 1$, let us set $|\xi|=\sqrt{\sum_{i=1}^{N} \xi_{i}^{2}}$. Let us remark that a growth condition like the following:

$$
a|\xi|^{p}+b \leq f(x, \eta, \xi), \quad p>1
$$

does not imply in general that a solution $\bar{u}$ of (4) belongs to $W^{1, p}(0,1)$. Actually $\bar{u}$ belongs to $W^{1, q}(0,1)$ for some $q$ such that $1 \leq q<p$.

In this work we also establish some regularity results for $\bar{u}$ like for instance the following: $\bar{u}^{\prime} \in L^{p}(0,1)$ or $\bar{u}^{\prime} \in$ $L^{\infty}(0,1)$. There are phenomena with memory effect. They are well known in the field of sciences and mathematical engineering. Besides, the intervention of these phenomena in economics, finance and environmental science is new and important. At time t the value of the control solution of such phenomena depends to its value at time $\mathrm{s}$ for any $s<t-c f$. for instance $[1,5,8,9,11,12,16]$. For such problems the goal is to optimize, for instance some functional like

$$
J(u, v)=\int_{0}^{1} f_{1}\left(t, u\left(\theta_{v}(t)\right), v(t)\right) \mathrm{d} t,
$$


where the control $(u, v)$ is submitted to some constraints such that $(u, v)$ belongs to some admissible control subset $\mathcal{U}_{a d}$ of some functional space and

$$
\left\{\begin{array}{l}
\frac{\mathrm{d} \theta_{v}(t)}{\mathrm{d} t}=g\left(t, \theta_{v}(t), v(t)\right), \quad t \in[0,1] \\
\theta_{v}(0)=\theta_{0}, 0 \leq \theta_{0} \leq 1, \quad \theta(t) \in[0,1] \text { for a.e. } t \in[0,1] .
\end{array}\right.
$$

Applied to this example our method gives the following result: Let $(\bar{u}, \bar{v})$ be the optimal solution of our problem. Then $(\bar{u}, \bar{v})$ satisfies an integrodifferential system having Dirac measures - cf. Sections 5.1 and 5.2. Finally in Section 6 we deal with the study of a financial market problem: the model of Jouini, Koel and Touzi [9,10]. As far as we know this problem is new by the fact that the delay function $\theta($.$) is an unknown parameter governed$ by a differential equation. It is a control problem - cf. also [7]. We show that the Euler-Lagrange equation is an integrodifferential one. In a regular framework, applied to this model, our result is more explicit than the result established in [9].

Let us point out that all the results of this work are valid in the framework where the state of the system and the control are in $\mathbb{R}^{N}, N \geq 1$. For convenience and to simplify the notations, our result is established in the case where $N=1$.

\section{Euler-Lagrange equation of problem (4)}

In the sequel we need to extend a result of [6]: Theorem 1, p. 96.

Proposition 2.1. Let $f$ and $g$ be such that $f \in W^{1, p}(0,1)$ and $g \in L^{q}(0,1)$, with $\frac{1}{p}+\frac{1}{q}=1, p \geq 1$. We suppose $g(x) \geq 0$ for a.e. $x$. Then we have the following formula:

$$
I=\int_{0}^{1} g(x)\left|f^{\prime}(x)\right| \mathrm{d} x=\int_{0}^{+\infty}\left(\sum_{x \in \partial_{\star} E_{y}} g(x)\right) \mathrm{d} y,
$$

where $E_{y}=\{t \in[0,1] / f(t)>y\}$ and $\partial_{\star} E_{y}$ stands for the reduced boundary of the level set of $E_{y}$.

Remark 2.1. For the definition of the reduced boundary of the level set $E_{y}$, we refer to [6], p. 194 .

Proof of Proposition 2.1. Let $\tilde{g}$ be such that $\tilde{g} \in L^{q}(0,1), \tilde{g}(x)=g(x)$ for a.e. $x$ and $\tilde{g}(x) \geq 0 \forall x$. It suffices to prove the result for $\tilde{g}$. For a.e. $y$ in $[0,1]$ let us set $\sigma(t)=H^{0}\left(\partial_{\star}\{t / f(t)>y\}\right)$, where $H^{0}$ stands for 0 -dimensional Haussdorf measure. Since $f$ belongs to $W^{1, p}(0,1)$, then we have for a.e. y $\sigma(y)<+\infty$. Let us set $E=\{y / \sigma(y)=+\infty\}$. We have $|E|=0$. Setting $F=\mathbb{R}^{+} \backslash E$, we have $\forall y \in F, \sigma(y)<+\infty$. For any $k \in \mathbb{N}$ and for any $x \in[0,1]$ let us set $\tilde{g}_{k}(x)=\inf \{k, \tilde{g}(x)\}$. Then $\tilde{g}_{k}($.$) belongs to L^{\infty}(0,1)$ and we have

$$
\int_{0}^{1} \tilde{g}(x)\left|f^{\prime}(x)\right| \mathrm{d} x \geq I_{k}=\int_{0}^{1} \tilde{g}_{k}(x)\left|f^{\prime}(x)\right| \mathrm{d} x=\int_{0}^{+\infty}\left(\sum_{x \in \partial_{\star} E_{y}} \tilde{g}_{k}(x)\right) \mathrm{d} y .
$$

For any $k \in \mathbb{N}$ and for any $y$ let us set $\psi_{k}(y)=\sum_{x \in \partial_{\star} E_{y}} \tilde{g}_{k}(x)$, the sequence $k \mapsto \psi_{k}($.$) is an increasing sequence$ of functions; thus by the monotonous convergence theorem, we obtain:

$$
\int_{0}^{+\infty} \psi_{k}(y) \mathrm{d} y \longrightarrow \int_{0}^{+\infty} \sup _{k} \psi_{k}(y) \mathrm{d} y \leq \int_{0}^{1}\left|f^{\prime}(x)\right| \tilde{g}(x) \mathrm{d} x .
$$

Then let us consider the following function:

$$
\psi: \mathbb{R}^{+} \longrightarrow \mathbb{R}^{+} \cup\{+\infty\}, y \longrightarrow \psi(y)=\sum_{x \in \partial_{\star} E_{y}} \tilde{g}(x),
$$


and let us set $G=\{y / \psi(y)=+\infty\}$. Let us show that $\sup \psi_{k}(y)=\psi(y)$ for a.e. $y$. It is enough to show that $|G \cap F|=0$. For this let us set $H=\{y / \psi(y)<\infty\}$. Thus we have $H=\mathbb{R}^{+} \backslash G$ and $H=F \cap H=$ $\{y / \psi(y)<\infty\}$.

For any $y \in H$, we have $\psi(y) \geq \psi_{k}(y)$. Let $k_{0} \in \mathbb{N}$ be such that $k_{0}>\psi(y)$. Then we have

$$
\forall k \geq k_{0}, \quad \psi_{k}(y)=\psi_{k_{0}}(y)
$$

i.e.

$$
\text { for any } k \geq k_{0}, \quad \psi(y) \geq \psi_{k}(y)=\psi_{k_{0}}(y) \quad \forall y \in H .
$$

Since the cardinal $\#\left(\partial_{\star} E_{y}\right)$ is finite we have

$$
\tilde{g}_{k_{0}}(x)=\tilde{g}(x) \quad \forall x \in \partial_{\star} E_{y} .
$$

(8) and (9) allow us to obtain

$$
\psi(y)=\sup _{k} \psi_{k}(y)=\lim _{k} \psi_{k}(y)=\psi_{k_{0}}(y)
$$

So we have finally

$$
\forall y \in H, \quad \psi(y)=\sup _{k} \psi_{k}(y) .
$$

Let now $y$ belonging to $F \cap G$, then we have: $\sum_{x \in \partial_{\star} E_{y}} \tilde{g}(x)=+\infty, \tilde{g}(x) \geq 0 \forall x \in \partial_{\star} E_{y}, \#\left(\partial_{\star} E_{y}\right)<+\infty$. So there exists $x_{0} \in \partial_{\star} E_{y}$ such that $\tilde{g}\left(x_{0}\right)=+\infty$. Hence we obtain

$$
\forall k \geq k_{0}, \quad \tilde{g}_{k}\left(x_{0}\right)=k .
$$

It follows that

$$
k=\tilde{g}_{k}\left(x_{0}\right) \leq \psi_{k}(y)=\sum_{x \in \partial_{\star} E_{y}} \tilde{g}_{k}(x) \leq \sum_{x \in \partial_{\star} E_{y}} \tilde{g}(x)=\psi(y)=+\infty,
$$

i.e. $\lim _{k \rightarrow+\infty} \psi_{k}(y)=+\infty$. Hence for any $y$ belonging to $F \cap G$ we have $\lim _{k \rightarrow+\infty} \psi_{k}(y)=+\infty$. So $\forall y \in$ $F \cap G, \sup _{k} \psi_{k}(y)=+\infty$. As we have

$$
\int_{0}^{+\infty} \sup _{k} \psi_{k}(y) \mathrm{d} y \leq \int_{0}^{1} \tilde{g}(x)\left|f^{\prime}(x)\right| \mathrm{d} x<+\infty,
$$

it follows that $\sup _{k} \psi_{k}(y)<+\infty$ for a.e. $y$ i.e. $|F \cap G|=0$. And it follows $\sup _{k} \psi_{k}(y)=\psi(y)$ for a.e. $y$. Finally we have

$$
\int_{0}^{1} \tilde{g}_{k}(x)\left|f^{\prime}(x)\right| \mathrm{d} x=\int_{0}^{+\infty} \psi_{k}(y) \mathrm{d} y .
$$

Passing to the limit in (13) as $k$ goes to infinity, we obtain

$$
\int_{0}^{1} \tilde{g}(x)\left|f^{\prime}(x)\right| \mathrm{d} x=\int_{0}^{+\infty} \psi(y) \mathrm{d} y=\int_{0}^{+\infty}\left(\sum_{x \in \partial_{\star} E_{y}} \tilde{g}(x)\right) \mathrm{d} y .
$$

Remark 2.2. The result is still true for scalar functions $u: \Omega \longrightarrow \mathbb{R}, \Omega \subset \mathbb{R}^{n}$ with $n>1$.

As a consequence of Proposition 2.1, it follows:

Proposition 2.2. Let $q$ be such that $1 \leq q<+\infty$ and let the following assumptions hold:

(i) $\theta:[0,1] \longrightarrow \mathbb{R}$ is an absolutely continuous function such that $\left|\theta^{\prime}().\right|$ belongs to $L^{q}(0,1)$;

(ii) $g$ belongs to $L^{q^{\prime}}(0,1)$, with $\frac{1}{q}+\frac{1}{q^{\prime}}=1$. 
Then we have:

$$
I=\int_{0}^{1} g(x)\left|\theta^{\prime}(x)\right| \mathrm{d} x=\int_{\mathbb{R}} h(x)\left|\theta^{\prime}(x)\right| \mathrm{d} x=\int_{\mathbb{R}}\left(\sum_{t \in \theta^{-1}(y)} g(t)\right) \mathrm{d} y,
$$

where $h=g \mathcal{X}_{[0,1]}$, and for any measurable subset $E$ of $[0,1] \mathcal{X}_{E}$ stands for the characteristic function of E.

Remark 2.3. The formula (14) is established in [6] under the assumptions that $g \in L^{1}(0,1)$ and $\theta$ is a Lipschitz function. The proof of [6] adapts to prove Proposition 2.2, using Proposition 2.1.

The Proposition 3 of [6], p. 119, is still valid under the following assumptions:

(i) $\theta:[0,1] \longrightarrow \mathbb{R}$ is an absolutely continuous function such that:

$$
\left|\theta^{\prime}(.)\right| \in L^{q}(0,1),\left|\theta^{\prime}(x)\right| \geq \theta_{0} \text { a.e. } x \in(0,1) \text { where } \theta_{0} \text { is a positive constant; }
$$

(ii) $g$ belongs to $L^{q^{\prime}}(0,1)$, with $\frac{1}{q}+\frac{1}{q^{\prime}}=1$.

Indeed, applying Proposition 2.2 we obtain:

$$
\begin{aligned}
\int_{\{x / \theta(x)>t\}} g(x) \mathrm{d} x=\int_{\{x / \theta(x>t\}} \mathcal{X}_{[0,1]}(x) g(x) \mathrm{d} x & =\int_{t}^{+\infty}\left(\sum_{x \in \theta^{-1}(s)} \mathcal{X}_{[0,1]}(x) \frac{g(x)}{\left|\theta^{\prime}(x)\right|}\right) \mathrm{d} s \\
& =\int_{t}^{+\infty}\left(\sum_{x \in \theta^{-1}(s)} \frac{g(x)}{\left|\theta^{\prime}(x)\right|}\right) \mathrm{d} s .
\end{aligned}
$$

And clearly it follows:

$$
\frac{\mathrm{d}}{\mathrm{d} t}\left[\int_{\{x / \theta(x)>t\}} g(x) \mathrm{d} x\right]=-\sum_{x \in \theta^{-1}(t)} \frac{g(x)}{\left|\theta^{\prime}(x)\right|}
$$

which is established in [6].

Proposition 2.3. Let $r$ and $q$ be such that $1 \leq r, q<+\infty$ and let the following assumptions hold:

(i) $\theta:[0,1] \longrightarrow \mathbb{R}$ is an absolutely continuous function such that:

$$
\left|\theta^{\prime}(.)\right| \in L^{r}(0,1), \frac{1}{\left|\theta^{\prime}(.)\right|} \in L^{\delta}(0,1)
$$

(ii) $f \in L^{q}(0,1)$, with $\frac{1}{\delta}=1-\left(\frac{1}{r}+\frac{1}{q}\right)$.

Then we have:

$$
\begin{gathered}
\int_{0}^{1}|f(x)| \mathrm{d} x=\int_{\mathbb{R}}\left[\sum_{x \in \theta^{-1}(s)} \frac{|f(x)|}{\left|\theta^{\prime}(x)\right|}\right] \mathrm{d} s \\
F(t)=\int_{\{x / \theta(x)>t\}}|f(x)| \mathrm{d} x=\int_{t}^{\infty}\left[\sum_{x \in \theta^{-1}(s)} \frac{|f(x)|}{\left|\theta^{\prime}(x)\right|}\right] \mathrm{d} s \\
\frac{\mathrm{d} F(t)}{\mathrm{d} t}=-\sum_{x \in \theta^{-1}(t)} \frac{|f(x)|}{\left|\theta^{\prime}(x)\right|} \text { a.e. } t \in \mathbb{R} .
\end{gathered}
$$

\section{Remark 2.4.}

(1) By (15) the derivative $\frac{\mathrm{d} F(t)}{\mathrm{d} t}$ belongs to $L^{1}(\mathbb{R})$. So it is also the distributional derivative of $F$.

(2) Under the same assumptions as in Proposition 2.3 the result is also valid for $F(t)=\int_{\{x / \theta(x)>t\}} f(x) \mathrm{d} x$. 
Proof of Proposition 2.3. We can assume that $f$ is positive, since we could otherwise consider $f^{+}()=.\sup (0, f()$. and $f^{-}()=.\sup (0,-f()$.$) instead of f$. We have:

$$
I=\int_{0}^{1} f(x) \mathrm{d} x=\int_{\mathbb{R}} \mathcal{X}_{[0,1]}(x) f(x) \mathrm{d} x=\int_{\mathbb{R}} \mathcal{X}_{[0,1]}(x) \frac{f(x)}{\left|\theta^{\prime}(x)\right|}\left|\theta^{\prime}(x)\right| \mathrm{d} x .
$$

Since $\frac{1}{\mid \theta^{\prime}(.)}$ belongs to $L^{\delta}(0,1)$ and $f$ belongs to $L^{q}(0,1)$, by Hölder the function $\frac{f(.)}{\left|\theta^{\prime}(.)\right|}$ belongs to $L^{\mu}(0,1)$ with $\frac{1}{\mu}=\frac{1}{\delta}+\frac{1}{q}$. Since $\frac{1}{\delta}=1-\left(\frac{1}{r}+\frac{1}{q}\right)$ we have $\frac{1}{\mu}+\frac{1}{r}=1$. Using Proposition 2.2 we obtain (15):

$$
I=\int_{\mathbb{R}}\left[\sum_{x \in \theta^{-1}(s)} \frac{f(x)}{\left|\theta^{\prime}(x)\right|}\right] \mathrm{d} s .
$$

Using the same arguments as in the proof of (15), we obtain (16):

$$
F(t)=\int_{\{x / \theta(x)>t\}}|f(x)| \mathrm{d} x=\int_{t}^{\infty}\left[\sum_{x \in \theta^{-1}(s)} \frac{|f(x)|}{\left|\theta^{\prime}(x)\right|}\right] \mathrm{d} s .
$$

The formula (17) follows from (16).

The following question is natural. Let us consider the function $F(t)=\int_{\{x / \theta(x)>t\}} f(x) \mathrm{d} x$. Under what conditions the following function:

$$
\frac{\mathrm{d} F(t)}{\mathrm{d} t}=-\sum_{x \in \theta^{-1}(t)} \frac{f(x)}{\left|\theta^{\prime}(x)\right|}
$$

belongs to $L^{\lambda}(0,1)$ for some suitable real number $\lambda$ such that $1 \leq \lambda \leq q$ ?

Proposition 2.4 will allow us to answer to this important question.

Before considering Proposition 2.4, let us recall the following classical result.

Lemma 2.1. Let $\left(a_{i}\right)_{i=1}^{k}$ be a finite sequence of real numbers and $\lambda$ be a real number such that $\lambda \geq 1$. Then we have

$$
\left(\sum_{i=1}^{k}\left|a_{i}\right|\right)^{\lambda} \leq k^{\lambda-1} \sum_{i=1}^{k}\left|a_{i}\right|^{\lambda} .
$$

Proposition 2.4. Let $\rho$ and $q$ be such that $1 \leq \rho, q<+\infty$ and let the following assumptions hold:

(i) $\theta:[0,1] \longrightarrow \mathbb{R}$ is an absolutely continuous function such that: $\left|\theta^{\prime}().\right|$ belongs to $L^{\rho}(0,1), \frac{1}{\left|\theta^{\prime}(.)\right|}$ belongs to $L^{\mu}(0,1)$ and there exists a positive constant $C_{0}$ such that $\operatorname{card}\left(\theta^{-1}(s)\right) \leq C_{0}$ for a.e. s;

(ii) $f$ belongs to $L^{q}(0,1)$, with $\frac{1}{\rho}+\frac{1}{q}+\frac{1}{\mu} \leq 1$, then we have:

$$
\frac{\mathrm{d} F(s)}{\mathrm{d} s}=k(s)=-\sum_{x \in \theta^{-1}(s)} \frac{f(x)}{\left|\theta^{\prime}(x)\right|} \text { belongs to } L^{\lambda}(0,1),
$$

where

and

$$
\lambda=\frac{(\rho-1) q \mu}{\rho(q+\mu)}, 1 \leq \lambda \leq q
$$

$$
F(s)=\int_{s}^{\infty}\left[\sum_{x \in \theta^{-1}(t)} \frac{f(x)}{\left|\theta^{\prime}(x)\right|}\right] \mathrm{d} t .
$$


Proof. Considering Proposition 2.3 it is enough to show that $|k(.)|^{\lambda}$ belongs to $L^{1}(0,1)$. We have:

$$
|k(s)|^{\lambda}=\left|\sum_{x \in \theta^{-1}(s)} \frac{f(x)}{\left|\theta^{\prime}(x)\right|}\right|^{\lambda} \text { for a.e. s. }
$$

Using Lemma 2.1 and the fact that $\operatorname{card}\left(\theta^{-1}(s)\right) \leq C_{0}$ for a.e. $s$ we obtain:

$$
|k(.)|^{\lambda} \leq C \sum_{x \in \theta^{-1}(s)}\left[\frac{|f(x)|^{\lambda}}{\left|\theta^{\prime}(x)\right|^{\lambda-1}} \frac{1}{\left|\theta^{\prime}(x)\right|}\right] \text { for a.e. } s
$$

where $C$ is a suitable constant depending on $C_{0}$ and on $\lambda$. Let us set

$$
m(x)=\frac{|f(x)|^{\lambda}}{\left|\theta^{\prime}(x)\right|^{\lambda-1}} \text { for a.e. } x .
$$

Then we have:

$$
|k(.)|^{\lambda} \leq C \sum_{x \in \theta^{-1}(s)} \frac{m(x)}{\left|\theta^{\prime}(x)\right|} \text { for a.e. } s .
$$

Let us write:

$$
\int_{0}^{1} m(x) \mathrm{d} x=\int_{0}^{1} \frac{m(x)}{\left|\theta^{\prime}(x)\right|}\left|\theta^{\prime}(x)\right| \mathrm{d} x .
$$

In order to apply Proposition 2.2 to the left hand side of the equality (20), it is enough to choose $\lambda$ such that the function $\frac{|f(x)|^{\lambda}}{\left|\theta^{\prime}(x)\right|^{\lambda}}$ belongs to $L^{\rho^{\prime}}(0,1)$ with $\frac{1}{\rho}+\frac{1}{\rho^{\prime}}=1$. Hence $\frac{|f(x)|}{\left|\theta^{\prime}(x)\right|}$ belongs to $L^{\lambda \rho^{\prime}}(0,1)$, where $\frac{1}{q}+\frac{1}{\mu}=\frac{1}{\lambda \rho^{\prime}}$ i.e. $\frac{\rho^{\prime}}{q}+\frac{\rho^{\prime}}{\mu}=\frac{1}{\lambda}$. Since $\rho^{\prime}=\frac{\rho}{\rho-1}$, we have:

$$
\lambda=\frac{(\rho-1) q \mu}{\rho(\mu+q)}
$$

It is easy to see that $\lambda \leq q$. Let us show that $\lambda \geq 1$. Since $\lambda=\frac{(\rho-1) q \mu}{\rho(\mu+q)}$, it is enough to show $\frac{(\rho-1) q \mu}{\rho(\mu+q)} \geq 1$ which can write $\frac{1}{\rho}+\frac{1}{q}+\frac{1}{\mu} \leq 1$. It is indeed the case by assumption. Therefore we have $1 \leq \lambda \leq q$. Moreover from (21) we obtain:

$$
\frac{1}{\rho}+\frac{\lambda}{\mu}+\frac{\lambda}{q}=1
$$

Under the condition (21) and using (19), we obtain:

$$
\int_{0}^{1}|k(s)|^{\lambda} \mathrm{d} s \leq C \int_{\mathbb{R}} \sum_{x \in \theta^{-1}(s)} \frac{m(x)}{\left|\theta^{\prime}(x)\right|} \mathrm{d} s=\int_{0}^{1} m(x) \mathrm{d} x<\infty,
$$

i.e.

$$
k(s)=\frac{\mathrm{d}}{\mathrm{d} s} \int_{s}^{\infty}\left[\sum_{x \in \theta^{-1}(t)} \frac{f(x)}{\left|\theta^{\prime}(x)\right|}\right] \mathrm{d} t \in L^{\lambda}(0,1) .
$$




\section{Remark 2.5.}

(1) If $\frac{1}{\rho}+\frac{1}{\mu}+\frac{1}{q}=1$, then we have $\lambda=1$.

(2) If $\left|\theta^{\prime}\right|$ and $\frac{1}{\left|\theta^{\prime}\right|}$ belong to $L^{\infty}(0,1)$, then $\lambda=q$. It is the case when $\theta$ satifies the following assumption: $0<\theta_{0} \leq\left|\theta^{\prime}(x)\right| \leq k$ for a.e. $x$.

Corollary 2.1. Let $q$ and $\nu$ be such that $1 \leq q<+\infty, 1 \leq \nu<+\infty$ and let the following assumptions hold:

(i) $\theta:[0,1] \longrightarrow \mathbb{R}$ is an absolutely continuous function such that:

$$
\left|\theta^{\prime}(.)\right| \in L^{\rho}(0,1), \frac{1}{\left|\theta^{\prime}(.)\right|} \in L^{\mu}(0,1), \mu \geq 1 ;
$$

(ii) $\operatorname{card}\left(\theta^{-1}(s)\right) \in L^{\nu}(\mathbb{R}), \nu \geq 1$;

(iii) $f \in L^{q}(0,1)$;

(iv) $\frac{1}{\rho}+\frac{1}{q}+\frac{1}{\mu} \leq 1$;

(v) $\frac{1}{\mu}+\frac{1}{\nu}+\frac{1}{\rho}+\frac{1}{q}-\frac{1}{\nu}\left(\frac{1}{q}+\frac{1}{\mu}+\frac{1}{\rho}\right) \leq 1$.

Then the function

$$
k(s)=\frac{\mathrm{d} F(s)}{\mathrm{d} s}=-\sum_{x \in \theta^{-1}(s)} \frac{f(x)}{\left|\theta^{\prime}(x)\right|} \text { belongs to } L^{\Lambda}(0,1),
$$

where

$$
\Lambda=\frac{q \mu \nu(\rho-1)}{(\rho-1) q \mu+\nu \rho(\mu+q)-\rho(\mu+q)} \text { and } F(s)=\int_{\{x / \theta(x)>s\}} f(x) \mathrm{d} x .
$$

Proof. Let $\lambda \geq 1$ be some real number to be determined later. By Lemma 2.1, we have:

$$
|k(s)|^{\lambda} \leq\left[\operatorname{card}\left(\theta^{-1}(s)\right)\right]^{\lambda-1} \sum_{x \in \theta^{-1}(s)} \frac{m(x)}{\left|\theta^{\prime}(x)\right|}
$$

where $m(x)=\frac{|f(x)|}{\left|\theta^{\prime}(x)\right|^{\lambda-1}}$ for a.e. $x$. Hence we obtain

$$
\left(\frac{|k(s)|}{\left[\operatorname{card}\left(\theta^{-1}(s)\right)\right]^{\frac{\lambda-1}{\lambda}}}\right)^{\lambda} \leq \sum_{x \in \theta^{-1}(s)} \frac{m(x)}{\left|\theta^{\prime}(x)\right|}
$$

Using Proposition 2.4 we see that the function $h(s)=\frac{|k(s)|}{\left[\operatorname{card}\left(\theta^{-1}(s)\right)\right]^{\frac{\lambda-1}{\lambda}}}$ belongs to $L^{\lambda}(0,1)$, where $\lambda$ is given by:

$$
\lambda=\frac{(\rho-1) q \mu}{\rho(\mu+q)},
$$

which satisfies $\lambda \geq 1$ by (iv). Since $\operatorname{card}\left(\theta^{-1}().\right)$ belongs to $L^{\nu}(\mathbb{R})$, we have

$$
\left[\operatorname{card}\left(\theta^{-1}(s)\right)\right]^{\frac{\lambda-1}{\lambda}} \in L^{\frac{\nu \lambda}{\lambda-1}}(0,1)
$$

Therefore the function $|k(s)|=h(s)\left[\operatorname{card}\left(\theta^{-1}(s)\right)\right]^{\frac{\lambda-1}{\lambda}}$ belongs to $L^{\Lambda}(0,1)$, with $\Lambda$ satisfying the following equality:

$$
\frac{1}{\Lambda}=\frac{1}{\lambda}+\frac{\lambda-1}{\nu \lambda}
$$


The relations (22) and (23) give

$$
\Lambda=\frac{q \mu \nu(\rho-1)}{(\rho-1) q \mu+\nu \rho(\mu+q)-\rho(\mu+q)} .
$$

By assumption (v), we show that $\Lambda \geq 1$. Indeed, to show that $\Lambda \geq 1$, it is enough to prove that:

$$
\frac{(\rho-1) q \mu+\nu \rho(\mu+q)-\rho(\mu+q)}{q \mu \nu(\rho-1)} \leq 1
$$

i.e.

$$
\frac{1}{\mu}+\frac{1}{\nu}+\frac{1}{\rho}+\frac{1}{q}-\frac{1}{\nu}\left(\frac{1}{q}+\frac{1}{\mu}+\frac{1}{\rho}\right) \leq 1
$$

It is indeed the case by assumption. Hence $\Lambda \geq 1$.

\section{Remark 2.6.}

(1) If $\frac{1}{\rho}+\frac{1}{\mu}+\frac{1}{q}=1$, then $\lambda=1$ and by equation (23) we obtain $\Lambda=1$.

(2) If $\left|\theta^{\prime}\right|$ and $\frac{1}{\left|\theta^{\prime}\right|}$ belong to $L^{\infty}(0,1)$, then $\lambda=q$ and by equation (23) we obtain $\Lambda=\frac{q \nu}{\nu+q-1}=$ $\left[\frac{1}{q}+\frac{1}{q \nu}-\frac{1}{\nu}\right]^{-1}$

(3) If $\nu=+\infty$, then $\Lambda=\lambda=\frac{(\rho-1) q \mu}{\rho(\mu+q)}$ from Proposition 2.4.

Let us now establish the Euler-Lagrange equation of the problem (4) where the functional space is the following one:

$$
W=\left\{v \in L^{1}(0,1) / v^{\prime} \in L^{p}\left((0,1), \frac{\mathrm{d} \mu_{\varphi}}{\mathrm{d} m}\right), v(0)=v(1)=0\right\}
$$

$v^{\prime}$ stands for the distributional derivative of $v$ and $\frac{\mathrm{d} \mu_{\varphi}}{\mathrm{d} m}$ is the Radon-Nikodym derivative of the measure $\mu_{\varphi}$ defined by: for any measurable $e \subset[0,1], \mu_{\varphi}(e)=\left|\varphi^{-1}(e)\right|$. Let us recall that the problem (4) has a solution in $W \subset W_{0}^{1, q}(0,1)$ if we assume the following [15]: There exists two positive real numbers $\beta$ and $q, 1 \leq \beta<q<p$, such that $\frac{\mathrm{d} \mu_{\varphi}}{\mathrm{d} m}$ satifies: $\frac{\mathrm{d} \mu_{\varphi}}{\mathrm{d} m} \in L^{\frac{q}{q-\beta}}(0,1)$ and $\left(\frac{\mathrm{d} \mu_{\varphi}}{\mathrm{d} m}\right)^{-1} \in L^{\frac{q}{p-q}}(0,1)$. (See also [4] for an approach slightly different.)

In order to establish Euler-Lagrange equation of the problem (4), we need the following assumptions. Let $f$ be a function such that $f:(x, \eta, \xi) \in[0,1] \times \mathbb{R}^{N} \times \mathbb{R}^{N} \longrightarrow f(x, \eta, \xi) \in \mathbb{R}$, differentiable with respect to the two last arguments $(\eta, \xi)$ for a.e. $x$, convex with respect to $\xi$.

All the results of this work are obtained for $N \geq 1$. To simplify we present our ideas in the case $N=1$. Let the following assumptions hold:

$$
\left\{\begin{array}{l}
a_{1}|\xi|^{p}+b_{1} \leq f(x, \eta, \xi) \leq a_{2}|\xi|^{p}+b_{2}, \quad a_{i}>0, \quad i=1,2, \quad p>1 \\
\left|\frac{\partial f}{\partial \eta}(x, \eta, \xi)\right|,\left|\frac{\partial f}{\partial \xi}(x, \eta, \xi)\right| \leq a_{3}|\xi|^{p-1}+b_{3}, \quad a_{3}>0 .
\end{array}\right.
$$

For simplicity of the notations, let the deviations $\theta($.$) and \varphi($.$) be such that \theta([0,1])=[0,1]$ and $\varphi([0,1])=[0,1]$ with $\theta($.$) and \varphi($.$) absolutely continuous. As the function t \mapsto|\{s \in[0,1] / \theta(s)>t\}|$ is decreasing, there exists a family of real numbers $\left(t_{i}\right)_{i \in I} \subset[0,1]$, with $I$ empty, finite or countable such that:

$$
\left|\left\{s \in[0,1] / \theta(s)=t_{i}\right\}\right|>0, \quad \forall i \in I
$$


Let us also assume

$$
\left\{\begin{array}{l}
\left|\theta^{\prime}(.)\right| \in L^{\rho}(0,1), \frac{\mathcal{X}_{\theta}(.)}{\theta^{\prime}(.) \mid} \in L^{\mu}(0,1), \operatorname{card}\left(\theta^{-1}(.)\right) \in L^{\nu}(0,1) \\
\text { with } \frac{1}{\rho}+\frac{1}{p^{\prime}}+\frac{1}{\mu} \leq 1, \frac{1}{\mu}+\frac{1}{\nu}+\frac{1}{\rho}+\frac{1}{p^{\prime}}-\frac{1}{\nu}\left(\frac{1}{p^{\prime}}+\frac{1}{\mu}+\frac{1}{\rho}\right) \leq 1, \\
\frac{1}{p}+\frac{1}{p^{\prime}}=1,
\end{array}\right.
$$

where

$$
\begin{gathered}
\mathcal{X}_{\theta}(.)=\mathcal{X}_{E}(.) \text { with } E=\left\{s \in[0,1] / \theta(s) \neq t_{i} \quad \forall i \in I\right\}, \\
\left\{\begin{array}{l}
\left|\varphi^{\prime}(.)\right| \in L^{\rho^{\prime}}(0,1), \frac{1}{\left|\varphi^{\prime}(.)\right|} \in L^{\mu^{\prime}}(0,1), \operatorname{card}\left(\varphi^{-1}(.)\right) \in L^{\nu^{\prime}}(0,1) \\
\text { with } \frac{1}{\rho^{\prime}}+\frac{1}{p^{\prime}}+\frac{1}{\mu^{\prime}} \leq 1, \frac{1}{\mu^{\prime}}+\frac{1}{\nu^{\prime}}+\frac{1}{\rho^{\prime}}+\frac{1}{p^{\prime}}-\frac{1}{\nu^{\prime}}\left(\frac{1}{p^{\prime}}+\frac{1}{\mu^{\prime}}+\frac{1}{\rho^{\prime}}\right) \leq 1 .
\end{array}\right.
\end{gathered}
$$

The deviation $\varphi($.$) satisfies the following:$

For any measurable $e \subset[0,1]$ such that $|e|=0$, we have $\left|\varphi^{-1}(e)\right|=0$.

Theorem 2.1. Under the assumptions hypeul1), (27), (28) and (29) every solution $u \in W$ of the problem (4) satisfies the following equation:

$$
\begin{array}{r}
\frac{\partial}{\partial \eta}\left\langle\nu_{t}^{\theta}, f\left(., u(t), u^{\prime} \circ \varphi(.)\right)\right\rangle+\sum_{i \in I} \delta_{t_{i}} \int_{\left\{\theta=t_{i}\right\}} \frac{\partial f}{\partial \eta}\left(s, u \circ \theta(s), u^{\prime} \circ \varphi(s)\right) \mathrm{d} s-\frac{\mathrm{d}}{\mathrm{d} t}\left[\frac{\partial}{\partial \xi}\left\langle\mu_{t}^{\varphi}, f\left(., u \circ \theta(.), u^{\prime}(t)\right\rangle\right]=0\right. \\
\quad \text { in } \mathcal{D}_{t}^{\prime}(0,1), \quad(30)
\end{array}
$$

with the short notation $\left\{\theta=t_{i}\right\}=\left\{s \in[0,1] / \theta(s)=t_{i}\right\}$, and $\nu_{t}^{\theta}$ and $\mu_{t}^{\varphi}$ stand for two counting measures defined respectively by:

$$
\text { for a.e. } t\left\langle\nu_{t}^{\theta}, f\left(., u(t), u^{\prime} \circ \varphi(.)\right)\right\rangle=\sum_{x \in \theta^{-1}(t)} \mathcal{X}_{\theta}(x) \frac{f\left(x, u(t), u^{\prime} \circ \varphi(x)\right)}{\left|\theta^{\prime}(x)\right|}
$$

and

$$
\text { for a.e. } t\left\langle\mu_{t}^{\varphi}, f\left(., u \circ \theta(.), u^{\prime}(t)\right)\right\rangle=\sum_{x \in \varphi^{-1}(t)} \frac{f\left(x, u \circ \theta(x), u^{\prime}(t)\right)}{\left|\varphi^{\prime}(x)\right|} \text {. }
$$

Remark 2.7. Let us point out that existence results of the problem (4) in $W$ have been studied in [15] (cf. also $[4,5])$.

Proof. Let $u$ be a solution in $W$ of the problem (4) then we have:

$$
\forall \epsilon \in \mathbb{R}, \forall v \in \mathcal{D}(0,1) \quad J(u+\epsilon v)-J(u) \geq 0 .
$$

In order to establish our result, we start from the following equation which follows from the problem (4):

$$
\lim _{\epsilon \rightarrow 0} \frac{J(u+\epsilon v)-J(u)}{\epsilon}=0, \quad \forall v \in \mathcal{D}(0,1) .
$$

Next we underline in (31) the two following functions:

$$
\begin{gathered}
t \rightarrow \int_{\{x / \theta(x)>t\}} \mathcal{X}_{\theta}(x) \frac{\partial f}{\partial \eta}\left(x, u \circ \theta(x), u^{\prime} \circ \varphi(x)\right) \mathrm{d} x, \\
t \rightarrow \int_{\{x / \varphi(x)>t\}} \frac{\partial f}{\partial \xi}\left(x, u \circ \theta(x), u^{\prime} \circ \varphi(x)\right) \mathrm{d} x .
\end{gathered}
$$

And finaly we establish the result by studying the Sobolev regularity of these functions. Therefore we shall lead the proof in three steps. 
First step. By the regularity and the growth assumptions on $f$, using the Lebesgue theorem, we obtain the existence of the limit in (31), and thus we have the following equation

$$
\int_{0}^{1} \frac{\partial f}{\partial \eta}\left(x, u \circ \theta(x), u^{\prime} \circ \varphi(x)\right) v \circ \theta(x) \mathrm{d} x+\int_{0}^{1} \frac{\partial f}{\partial \xi}\left(x, u \circ \theta(x), u^{\prime} \circ \varphi(x)\right) v^{\prime} \circ \varphi(x) \mathrm{d} x=0 \quad \forall v \in \mathcal{D}(0,1) .
$$

Second step. Let us set $I_{1}=\int_{0}^{1} \frac{\partial f}{\partial \eta}\left(x, u \circ \theta(x), u^{\prime} \circ \varphi(x)\right) v \circ \theta(x) \mathrm{d} x$. Using (26), we can also rewrite $I_{1}$ in the following form:

$$
\begin{aligned}
I_{1}= & \sum_{i \in I} \int_{\left\{x \in[0,1] / \theta(x)=t_{i}\right\}} \frac{\partial f}{\partial \eta}\left(x, u \circ \theta(x), u^{\prime} \circ \varphi(x)\right) v\left(t_{i}\right) \mathrm{d} x \\
& +\int_{\left\{x \in[0,1] / \theta(x) \neq t_{i} \forall i \in I\right\}} \frac{\partial f}{\partial \eta}\left(x, u \circ \theta(x), u^{\prime} \circ \varphi(x)\right) v \circ \theta(x) \mathrm{d} x \quad \forall v \in \mathcal{D}(0,1) .
\end{aligned}
$$

Using the following remark in the second term of $I_{1}$ :

$$
v \circ \theta(x)=\int_{0}^{\theta(x)} v^{\prime}(t) \mathrm{d} t=\int_{\{t \in[0,1] / \theta(x) \geq t\}} v^{\prime}(t) \mathrm{d} t \quad \forall v \in \mathcal{D}(0,1),
$$

we obtain

$$
\begin{aligned}
\int_{\left\{x \in[0,1] / \theta(x) \neq t_{i} \forall i \in I\right\}} \frac{\partial f}{\partial \eta}\left(x, u \circ \theta(x), u^{\prime} \circ \varphi(x)\right) v \circ \theta(x) \mathrm{d} x & \\
& =\int_{\left\{x \in[0,1] / \theta(x) \neq t_{i} \forall i \in I\right\}} \frac{\partial f}{\partial \eta}\left(x, u \circ \theta(x), u^{\prime} \circ \varphi(x)\right)\left[\int_{\{t \in[0,1] / \theta(x) \geq t\}} v^{\prime}(t) \mathrm{d} t\right] \mathrm{d} x
\end{aligned}
$$

Let us set

and

$$
F(x, t)=\frac{\partial f}{\partial \eta}\left(x, u \circ \theta(x), u^{\prime} \circ \varphi(x)\right) v^{\prime}(t)
$$

$$
D=\left\{(x, t) \in[0,1] \times[0,1] / \theta(x) \geq t, \theta(x) \neq t_{i} \quad \forall i \in I\right\} .
$$

Let us show that $F(.,$.$) belongs to L^{1}([0,1] \times[0,1])$. By $(25)$, we have:

$$
\int_{[0,1] \times[0,1]}|F(x, t)| \mathrm{d} x \leq \int_{[0,1] \times[0,1]}\left[a_{2}\left|u^{\prime} \circ \varphi(x)\right|^{p}+\left|b_{2}\right|\right]\left|v^{\prime}(t)\right| \mathrm{d} x \mathrm{~d} t .
$$

But

$$
\int_{[0,1] \times[0,1]}\left[a_{2}\left|u^{\prime} \circ \varphi(x)\right|^{p}+\left|b_{2}\right|\right]\left|v^{\prime}(t)\right| \mathrm{d} x \mathrm{~d} t=\left\{\int_{0}^{1}\left[a_{2}\left|u^{\prime}(x)\right|^{p} \frac{\mathrm{d} \mu_{\varphi}}{\mathrm{d} m}(x)+\left|b_{2}\right|\right] \mathrm{d} x\right\}\left\{\int_{0}^{1}\left|v^{\prime}(t)\right| \mathrm{d} t\right\}
$$

thus we obtain

$$
\int_{[0,1] \times[0,1]}|F(x, t)| \mathrm{d} x \mathrm{~d} t<\infty, \text { since } u \in W \text { and } v \in \mathcal{D}(0,1) .
$$

And since $D \subseteq[0,1] \times[0,1]$, the claimed result follows:

$$
\int_{D}|F(x, t)| \mathrm{d} x \mathrm{~d} t<\infty
$$


Using Fubini's theorem we obtain:

$$
\begin{aligned}
\int_{\left\{x \in[0,1] / \theta(x) \neq t_{i} \forall i \in I\right\}} \frac{\partial f}{\partial \eta}\left(x, u \circ \theta(x), u^{\prime} \circ \varphi(x)\right)\left[\int_{\{t \in[0,1] / \theta(x) \geq t\}} v^{\prime}(t) \mathrm{d} t\right] \mathrm{d} x= \\
\int_{0}^{1} v^{\prime}(t)\left[\int_{\left\{x \in[0,1] / \theta(x)>t, \theta(x) \neq t_{i} \forall i \in I\right\}} \frac{\partial f}{\partial \eta}\left(x, u \circ \theta(x), u^{\prime} \circ \varphi(x)\right) \mathrm{d} x\right] \mathrm{d} t .
\end{aligned}
$$

But we have

$$
\int_{\left\{x \in[0,1] / \theta(x)>t, \theta(x) \neq t_{i} \forall i \in I\right\}} \frac{\partial f}{\partial \eta}\left(x, u \circ \theta(x), u^{\prime} \circ \varphi(x)\right) \mathrm{d} x=\int_{\{x \in[0,1] / \theta(x)>t\}} \mathcal{X}_{\theta}(x) \frac{\partial f}{\partial \eta}\left(x, u \circ \theta(x), u^{\prime} \circ \varphi(x)\right) \mathrm{d} x .
$$

Thus we can finally rewrite $I_{1}$ in the following form:

$$
\begin{aligned}
I_{1}= & \sum_{i \in I} \int_{\left\{x \in[0,1] / \theta(x)=t_{i}\right\}} \frac{\partial f}{\partial \eta}\left(x, u \circ \theta(x), u^{\prime} \circ \varphi(x)\right)\left\langle\delta_{t_{i}}, v\right\rangle \mathrm{d} x \\
& +\int_{0}^{1} v^{\prime}(t)\left[\int_{\{x \in[0,1] / \theta(x)>t\}} \mathcal{X}_{\theta}(x) \frac{\partial f}{\partial \eta}\left(x, u \circ \theta(x), u^{\prime} \circ \varphi(x)\right) \mathrm{d} x\right] \mathrm{d} t .
\end{aligned}
$$

Now let us consider the second part of (32):

$$
I_{2}=\int_{0}^{1} \frac{\partial f}{\partial \xi}\left(x, u \circ \theta(x), u^{\prime} \circ \varphi(x)\right) v^{\prime} \circ \varphi(x) \mathrm{d} x
$$

and let us remark that

$$
v^{\prime} \circ \varphi(x)=\int_{0}^{\varphi(x)} v^{\prime \prime}(t) \mathrm{d} t \quad \forall v \in \mathcal{D}(0,1)
$$

Using the same arguments as for $I_{1}$, we obtain:

$$
I_{2}=\int_{0}^{1} v^{\prime \prime}(t)\left[\int_{\{x \in[0,1] / \varphi(x)>t\}} \frac{\partial f}{\partial \xi}\left(x, u \circ \theta(x), u^{\prime} \circ \varphi(x)\right) \mathrm{d} x\right] \mathrm{d} t .
$$

Finally (32) is equivalent to the following equation:

$$
\begin{aligned}
\sum_{i \in I} \int_{\left\{x \in[0,1] / \theta(x)=t_{i}\right\}} \frac{\partial f}{\partial \eta}\left(x, u \circ \theta(x), u^{\prime} \circ \varphi(x)\right)\left\langle\delta_{t_{i}}, v\right\rangle \mathrm{d} x & \\
+ & \int_{0}^{1} v^{\prime}(t)\left[\int_{\{x \in[0,1] / \theta(x)>t\}} \mathcal{X}_{\theta}(x) \frac{\partial f}{\partial \eta}\left(x, u \circ \theta(x), u^{\prime} \circ \varphi(x)\right) \mathrm{d} x\right] \mathrm{d} t \\
& +\int_{0}^{1} v^{\prime \prime}(t)\left[\int_{\{x \in[0,1] / \varphi(x)>t\}} \frac{\partial f}{\partial \xi}\left(x, u \circ \theta(x), u^{\prime} \circ \varphi(x)\right) \mathrm{d} x\right] \mathrm{d} t=0 \quad \forall v \in \mathcal{D}(0,1)
\end{aligned}
$$

Third step. Let us set

$$
H(t)=\int_{\{x \in[0,1] / \theta(x)>t\}} \mathcal{X}_{\theta}(x) \frac{\partial f}{\partial \eta}\left(x, u \circ \theta(x), u^{\prime} \circ \varphi(x)\right) \mathrm{d} x
$$

and

$$
G(t)=\int_{\{x \in[0,1] / \varphi(x)>t\}} \frac{\partial f}{\partial \xi}\left(x, u \circ \theta(x), u^{\prime} \circ \varphi(x)\right) \mathrm{d} x .
$$


Let us remark that by (27) and (28), $\theta($.$) and \varphi($.$) verify the assumptions of Corollary 2.1. Then using this$ corollary, we have

$$
\text { for a.e.t } \frac{\mathrm{d} H(t)}{\mathrm{d} t}=-\sum_{x \in \theta^{-1}(t)} \mathcal{X}_{\theta}(x) \frac{\frac{\partial f}{\partial \eta}\left(x, u(t), u^{\prime} \circ \varphi(x)\right)}{\left|\theta^{\prime}(x)\right|},
$$

and $\frac{\mathrm{d} H(t)}{\mathrm{d} t}$ belongs to $L^{\Lambda}$, where

$$
\Lambda=\frac{p^{\prime} \mu \nu(\rho-1)}{(\rho-1) p^{\prime} \mu+\nu \rho\left(\mu+p^{\prime}\right)-\rho\left(\mu+p^{\prime}\right)} .
$$

We also have

$$
\text { for a.e. } \frac{\mathrm{d} G(t)}{\mathrm{d} t}=-\sum_{x \in \varphi^{-1}(t)} \frac{\frac{\partial f}{\partial \xi}\left(x, u \circ \theta(x), u^{\prime}(t)\right)}{\left|\varphi^{\prime}(x)\right|},
$$

and $\frac{\mathrm{d} G(t)}{\mathrm{d} t}$ belongs to $L^{\Lambda^{\prime}}$, where

$$
\Lambda^{\prime}=\frac{p^{\prime} \mu^{\prime} \nu^{\prime}\left(\rho^{\prime}-1\right)}{\left(\rho^{\prime}-1\right) p^{\prime} \mu^{\prime}+\nu^{\prime} \rho^{\prime}\left(\mu^{\prime}+p^{\prime}\right)-\rho^{\prime}\left(\mu^{\prime}+p^{\prime}\right)}
$$

From (33), (34) and (35) we deduce the following equation

$$
\begin{aligned}
\sum_{i \in I} \delta_{t_{i}} \int_{\left\{x \in[0,1] / \theta(x)=t_{i}\right\}} \frac{\partial f}{\partial \eta}\left(x, u \circ \theta(x), u^{\prime} \circ \varphi(x)\right) \mathrm{d} x+\frac{\partial}{\partial \eta}\left\langle\nu_{t}^{\theta}, f\left(., u(t), u^{\prime} \circ \varphi(.)\right)\right\rangle & -\frac{\mathrm{d}}{\mathrm{d} t}\left[\frac{\partial}{\partial \xi}\left\langle\mu_{t}^{\varphi}, f\left(., u \circ \theta(.), u^{\prime}(t)\right)\right\rangle\right]=0 \text { in } \mathcal{D}_{t}^{\prime}(0,1),
\end{aligned}
$$

where

$$
\left\langle\nu_{t}^{\theta}, f\left(., u(t), u^{\prime} \circ \varphi(.)\right)\right\rangle=\sum_{x \in \theta^{-1}(t)} \mathcal{X}_{\theta}(x) \frac{f\left(x, u(t), u^{\prime} \circ \varphi(x)\right)}{\left|\theta^{\prime}(x)\right|} \quad \text { for a.e. } t \in[0,1]
$$

and

$$
\left\langle\mu_{t}^{\varphi}, f\left(., u \circ \theta(.), u^{\prime}(t)\right)\right\rangle=\sum_{x \in \varphi^{-1}(t)} \frac{f\left(x, u \circ \theta(x), u^{\prime}(t)\right)}{\left|\varphi^{\prime}(x)\right|} \quad \text { for a.e. } t \in[0,1] .
$$

\section{Remarks 2.1.}

(1) In the multidimensionnal case, putting $\eta=\left(\eta_{1}, \ldots, \eta_{N}\right), \xi=\left(\xi_{1}, \ldots, \xi_{N}\right), u \circ \theta=\left(u_{1} \circ \theta, \ldots, u_{N} \circ \theta\right)$, $u^{\prime} \circ \varphi=\left(u_{1}^{\prime} \circ \varphi, \ldots, u_{N}^{\prime} \circ \varphi\right)$, we obtain the following result: Under suitable assumptions as in Theorem 2.1 the Euler-Lagrange equation of our problem is

$\frac{\partial}{\partial \eta_{j}}\left\langle\nu_{t}^{\theta}, f\left(., u(t), u^{\prime}(\varphi()).\right)\right\rangle+\sum_{i \in I} \delta_{t_{i}} \int_{\left\{\theta=t_{i}\right\}} \frac{\partial f}{\partial \eta_{j}}\left(s, u(\theta(s)), u^{\prime}(\varphi(s))\right) \mathrm{d} s-\frac{\mathrm{d}}{\mathrm{d} t}\left[\frac{\partial}{\partial \xi_{j}}\left\langle\mu_{t}^{\varphi}, f\left(., u(\theta()),. u^{\prime}(t)\right)\right\rangle\right]=0$ for any $j=1,2, \ldots, N$.

(2) It is clear that we can choose $\theta=\left(\theta_{1}, \ldots, \theta_{N}\right), \varphi=\left(\varphi_{1}, \ldots, \varphi_{N}\right), u \circ \theta=\left(u_{1} \circ \theta_{1}, \ldots, u_{N} \circ \theta_{N}\right)$, $u^{\prime} \circ \varphi=\left(u_{1}^{\prime} \circ \varphi_{1}, \ldots, u_{N}^{\prime} \circ \varphi_{N}\right)$. 


\section{Application to the Regularity of solutions of the problem (4)}

In general the solutions of problem (4) do not belong to $W_{0}^{1, p}(0,1)$ if we consider the following growth condition: $a|\xi|^{p}+b \leq f(x, \eta, \xi), p>1$. They belong to $W_{0}^{1, q}(0,1)$ for some real number $q$ such that $1<q<p$. To obtain more regularity from the Euler-Lagrange equation (30) stated in Theorem 2.1, we have to delete Dirac measures. Therefore, we assume that the deviation $\theta($.$) has the following property:$

$$
|\{s \in[0,1] / \theta(s)=t\}|=0, \quad \forall t .
$$

Corollary 3.1. Let the following assumptions hold:
(i) $\left\{\begin{array}{l}a_{1}|\xi|^{p}+b_{1} \leq f(x, \eta, \xi) \leq a_{2}|\xi|^{p}+b_{2}, \quad a_{i}>0, \quad i=1,2, \quad p>1 \\ \left|\frac{\partial f}{\partial \eta}(x, \eta, \xi)\right|,\left|\frac{\partial f}{\partial \xi}(x, \eta, \xi)\right| \leq a_{3}|\xi|^{p-1}+b_{3}, \quad a_{3}>0 .\end{array}\right.$
(ii) $\left\{\begin{array}{l}\theta:[0,1] \longrightarrow[0,1] \text { is absolutely continuous such that: } \\ \left|\theta^{\prime}\right| \in L^{\rho}(0,1), \frac{1}{\left|\theta^{\prime}\right|} \in L^{\mu}(0,1), \operatorname{card}\left(\theta^{-1}(s)\right) \in L^{\nu}(0,1) \\ \text { with } \frac{1}{\rho}+\frac{1}{p^{\prime}}+\frac{1}{\mu} \leq 1, \frac{1}{\mu}+\frac{1}{\nu}+\frac{1}{\rho}+\frac{1}{p^{\prime}}-\frac{1}{\nu}\left(\frac{1}{p^{\prime}}+\frac{1}{\mu}+\frac{1}{\rho}\right) \leq 1, \\ \frac{1}{p}+\frac{1}{p^{\prime}}=1 .\end{array}\right.$
(iii) $\left\{\begin{array}{l}\varphi:[0,1] \longrightarrow[0,1] \text { is absolutely continuous such that: } \\ \left|\varphi^{\prime}\right| \in L^{\rho^{\prime}}(0,1), \frac{1}{\left|\varphi^{\prime}\right|} \in L^{\mu^{\prime}}(0,1), \operatorname{card}\left(\varphi^{-1}(s)\right) \in L^{\nu^{\prime}}(0,1) \\ \text { with } \frac{1}{\rho^{\prime}}+\frac{1}{p^{\prime}}+\frac{1}{\mu^{\prime}} \leq 1, \frac{1}{\mu^{\prime}}+\frac{1}{\nu^{\prime}}+\frac{1}{\rho^{\prime}}+\frac{1}{p^{\prime}}-\frac{1}{\nu^{\prime}}\left(\frac{1}{p^{\prime}}+\frac{1}{\mu^{\prime}}+\frac{1}{\rho^{\prime}}\right) \leq 1 . \\ \forall e \subset[0,1] \text { such that }|e|=0, \text { we have }\left|\varphi^{-1}(e)\right|=0 .\end{array}\right.$
(iv) $|\{s \in[0,1] / \theta(s)=t\}|=0, \quad \forall t$.

Then for every solution $u$ in $W \subseteq W_{0}^{1, q}(0,1)$ of the problem (4), we have:

If $\left|\varphi^{\prime}(t)\right| \leq C_{0}$ for a.e. $t$ then $u^{\prime} \in L^{\infty}(0,1)$.

If $k(t)=\left[\sum_{x \in \varphi^{-1}(t)} \frac{1}{\left|\varphi^{\prime}(x)\right|}\right]^{-1} \in L^{p}(0,1)$, then $u^{\prime} \in L^{p}(0,1)$ if $2<q<p<\infty$,
and $u^{\prime} \in L^{\frac{q p^{2}}{p+q}}(0,1)$ if $1 \leq q<p<\infty$.
\[ \text { And if } k(t)=\left[\sum_{x \in \varphi^{-1}(t)} \frac{1}{\left|\varphi^{\prime}(x)\right|}\right]^{-1} \in L^{\delta}(0,1) \text {, then } u^{\prime} \in L^{\frac{\delta p q}{\delta+q}}(0,1) \text { if } q \leq \delta \leq 2<p<\infty \text {. } \]

To prove Corollary 3.1, the following classical proposition will be useful.

Proposition 3.1. Let $p$ and $f$ be such that $1<p<+\infty$ and $f:[0,1] \times \mathbb{R} \times \mathbb{R} \longrightarrow \mathbb{R},(x, \eta, \xi) \longrightarrow f(x, \eta, \xi)$, convex with respect to $\xi$ such that:

$$
a_{1}|\xi|^{p}+b_{1} \leq f(x, \eta, \xi) \leq a_{2}|\xi|^{p}+b_{2}, \quad a_{i}>0, \quad i=1,2, \quad \forall \eta \in \mathbb{R}, \quad \forall \xi \in \mathbb{R}, \text { a.e. } x \in[0,1] .
$$

Then there exist two positive constants $\alpha$ and $\gamma$ such that:

$$
\xi \frac{\partial f}{\partial \xi}(x, \eta, \xi) \geq \alpha|\xi|^{p}-\gamma, \quad \forall \eta \in \mathbb{R}, \quad \forall \xi \in \mathbb{R}, \text { a.e. } x \in[0,1] .
$$

Proof of Corollary 3.1. Let $u$ be a solution of the problem (4). Under the hypotheses (i) to (iv), $u$ satisfies the following equation:

$$
\frac{\partial}{\partial \eta}\left\langle\nu_{t}^{\theta}, f\left(., u(t), u^{\prime} \circ \varphi(.)\right)\right\rangle-\frac{\mathrm{d}}{\mathrm{d} t}\left[\frac{\partial}{\partial \xi}\left\langle\mu_{t}^{\varphi}, f\left(., u \circ \theta(.), u^{\prime}(t)\right\rangle\right]=0 \text { in } \mathcal{D}_{t}^{\prime}(0,1),\right.
$$

where $\frac{\partial}{\partial \eta}\left\langle\nu_{t}^{\theta}, f\left(., u(t), u^{\prime} \circ \varphi().\right)\right\rangle$ stands for $\frac{\partial}{\partial \eta}\left\langle\nu_{t}^{\theta}, f\left(., \eta, u^{\prime} \circ \varphi(.)\right)\right\rangle_{\eta=u(t)}$. Thanks to Corollary 2.1 the two following functions $t \mapsto \frac{\partial}{\partial \eta}\left\langle\nu_{t}^{\theta}, f\left(., u(t), u^{\prime} \circ \varphi().\right)\right\rangle$ and $t \mapsto \frac{\partial}{\partial \xi}\left\langle\mu_{t}^{\varphi}, f\left(., u \circ \theta(),. u^{\prime}(t)\right)\right\rangle$ belong respectively to 
$L^{\Lambda}(0,1)$ and $L^{\Lambda^{\prime}}(0,1)$, where

$$
\Lambda=\frac{p^{\prime} \mu \nu(\rho-1)}{(\rho-1) p^{\prime} \mu+\nu \rho\left(\mu+p^{\prime}\right)-\rho\left(\mu+p^{\prime}\right)},
$$

and

$$
\Lambda^{\prime}=\frac{p^{\prime} \mu^{\prime} \nu^{\prime}\left(\rho^{\prime}-1\right)}{\left(\rho^{\prime}-1\right) p^{\prime} \mu^{\prime}+\nu^{\prime} \rho^{\prime}\left(\mu^{\prime}+p^{\prime}\right)-\rho^{\prime}\left(\mu^{\prime}+p^{\prime}\right)}
$$

From (37), this entails that:

$$
\left\langle\mu_{t}^{\varphi}, \frac{\partial f}{\partial \xi}\left(., u \circ \theta(.), u^{\prime}(t)\right)\right\rangle=\int_{0}^{t}\left\langle\nu_{s}^{\theta}, \frac{\partial f}{\partial \eta}\left(., u(s), u^{\prime} \circ \varphi(.)\right)\right\rangle \mathrm{d} s+C_{1} \text { for a.e. } t
$$

where $C_{1}$ is some constant. This implies that:

$$
\left\langle\mu_{t}^{\varphi}, u^{\prime}(t) \frac{\partial f}{\partial \xi}\left(., u \circ \theta(.), u^{\prime}(t)\right)\right\rangle=u^{\prime}(t) \int_{0}^{t}\left\langle\nu_{s}^{\theta}, \frac{\partial f}{\partial \eta}\left(., u(s), u^{\prime} \circ \varphi(.)\right)\right\rangle \mathrm{d} s+C_{1} u^{\prime}(t) \text { for a.e. } t \in(0,1)
$$

Since for almost every where $t, \mu_{t}^{\varphi}$ is a positive measure and since the function $s \mapsto\left\langle\nu_{s}^{\theta}, \frac{\partial f}{\partial \eta}\left(., u(s), u^{\prime} \circ \varphi().\right)\right\rangle$ belongs to $L_{s}^{r}(0,1)$, where

$$
r=\frac{p^{\prime} \mu \nu(\rho-1)}{(\rho-1) p^{\prime} \mu+\nu \rho\left(\mu+p^{\prime}\right)-\rho\left(\mu+p^{\prime}\right)} \geq 1,
$$

we obtain from Proposition 3.1 and equation (38):

$$
\left(\alpha\left|u^{\prime}(t)\right|^{p}-\gamma\right) \sum_{x \in \varphi^{-1}(t)} \frac{1}{\left|\varphi^{\prime}(x)\right|} \leq C\left|u^{\prime}(t)\right| \text { for a.e. } t \in(0,1),
$$

where $C$ stands for some constant. Let us prove the claimed result.

First case. If $\left|\varphi^{\prime}(t)\right| \leq C_{0}$ for a.e. $t$, then $u^{\prime}$ belongs to $L^{\infty}(0,1)$.

Indeed we have

$$
\sum_{x \in \varphi^{-1}(t)} \frac{1}{\left|\varphi^{\prime}(x)\right|} \geq \frac{\operatorname{card} \varphi^{-1}(t)}{C_{0}} \geq \frac{1}{C_{0}}
$$

since $\varphi([0,1])=[0,1]$ and $\operatorname{card} \varphi^{-1}(t) \geq 1 \forall t$. From (39) we obtain

$$
\left|u^{\prime}(t)\right|^{p} \leq C_{1}\left(\left|u^{\prime}(t)\right|+1\right) \text { for a.e. } t \in(0,1),
$$

where $C_{1}$ is some constant. We claim that (40) entails that $u^{\prime}$ belongs to $L^{\infty}(0,1)$. We argue by contradiction. If $u^{\prime} \notin L^{\infty}(0,1)$ then there exists $t_{n} \in[0,1]$ such that:

$$
\lim _{n \rightarrow+\infty}\left|u^{\prime}\left(t_{n}\right)\right|=+\infty
$$

From (40) we have:

$$
1 \leq C_{1}\left(\frac{1}{\left|u^{\prime}\left(t_{n}\right)\right|^{p-1}}+\frac{1}{\left|u^{\prime}\left(t_{n}\right)\right|^{p}}\right),
$$

this is a contradiction as $n$ goes to infinity, since $p>1$. This proves that $u^{\prime} \in L^{\infty}(0,1)$. 
Second case. If $k(t)=\left[\sum_{x \in \varphi^{-1}(t)} \frac{1}{\left|\varphi^{\prime}(x)\right|}\right]^{-1} \in L^{p}(0,1)$, with $2<q<p<\infty$, then $u^{\prime} \in L^{p}(0,1)$.

To prove this claimed result we start from inequality (39) that we integrate on the set $I_{n}=\{x \in[0,1] /$ $\left.\left|u^{\prime}(x)\right| \leq n\right\}$. We have a non decreasing sequence of measurables subsets $I_{n}$ of $[0,1]$ such that $\bigcup_{n} I_{n}=[0,1]$. From inequality (39) we have:

$$
\alpha\left|u^{\prime}(t)\right|^{p} \leq C\left|u^{\prime}(t)\right| k(t)+\gamma .
$$

From the existence result of the problem (4) in [15], there exists some real number $q, 2<q<p<+\infty$ such that $u^{\prime} \in L^{q}(0,1)$. Then $u^{\prime} \in L^{q^{\prime}}(0,1)$. This entails that $u^{\prime} \in L^{p^{\prime}}(0,1)$, where $\frac{1}{p}+\frac{1}{p^{\prime}}=1$. From inequality (41) we have:

$$
\alpha \int_{I_{n}}\left|u^{\prime}(t)\right|^{p} \mathrm{~d} t \leq C \int_{I_{n}}\left|u^{\prime}(t)\right| k(t) \mathrm{d} t+\gamma
$$

Hölder's inequality gives:

$$
\int_{I_{n}}\left|u^{\prime}(t)\right| k(t) \mathrm{d} t \leq\left(\int_{I_{n}}\left|u^{\prime}(t)\right|^{p^{\prime}} \mathrm{d} t\right)^{\frac{1}{p}}\left(\int_{I_{n}} k^{p}(t) \mathrm{d} t\right)^{\frac{1}{p}} .
$$

Thus we obtain from (42):

$$
\begin{aligned}
& \alpha \int_{I_{n}}\left|u^{\prime}(t)\right|^{p} \mathrm{~d} t \leq C\left(\int_{I_{n}}\left|u^{\prime}(t)\right|^{p^{\prime}} \mathrm{d} t\right)^{\frac{1}{p^{\prime}}}\left(\int_{I_{n}} k^{p}(t) \mathrm{d} t\right)^{\frac{1}{p}}+\gamma \\
& \quad \leq C_{2}\left[\left(\int_{I_{n}}\left|u^{\prime}(t)\right|^{p^{\prime}} \mathrm{d} t\right)^{\frac{1}{p^{\prime}}}+1\right] \leq C_{2}\left[\left(\int_{0}^{1}\left|u^{\prime}(t)\right|^{p^{\prime}} \mathrm{d} t\right)^{\frac{1}{p^{\prime}}}+1\right],
\end{aligned}
$$

where $C_{2}$ is some constant independent with respect to $n$. Then let us remark that there exists some constant $C_{3}$ such that:

$$
\forall n \quad a_{n}=\int_{I_{n}}\left|u^{\prime}(t)\right|^{p} \mathrm{~d} t \leq C_{3} .
$$

As $I_{n}$ is a nondecreasing sequence of subsets of $[0,1]$, the monotonous convergence theorem gives the following:

$$
\int_{0}^{1}\left|u^{\prime}(t)\right|^{p} \mathrm{~d} t \leq C_{3}
$$

So we have proved that $u^{\prime}$ belongs $L^{p}(0,1)$.

Third case. If $k(t)=\left[\sum_{x \in \varphi^{-1}(t)} \frac{1}{\left|\varphi^{\prime}(x)\right|}\right]^{-1} \in L^{p}(0,1)$, with $1 \leq q<p<\infty$, then $u^{\prime} \in L^{\frac{q p^{2}}{p+q}}(0,1)$.

As we have $1 \leq q<p<+\infty$, then thanks to the existence result of the problem (4), we obtain $u^{\prime} \in L^{q}(0,1)$. From (41) we have:

$$
\alpha^{\frac{p q}{p+q}} \int_{I_{n}}\left|u^{\prime}(t)\right|^{\frac{q p^{2}}{p+q}} \mathrm{~d} t \leq C_{4}\left(\int_{I_{n}}\left|u^{\prime}(t)\right|^{\frac{p q}{p+q}} k^{\frac{p q}{p+q}}(t) \mathrm{d} t+\gamma^{\frac{p q}{p+q}}\right) .
$$

Hölder's inequality shows that:

$$
\int_{I_{n}}\left|u^{\prime}(t)\right|^{\frac{p q}{p+q}} k^{\frac{p q}{p+q}}(t) \mathrm{d} t \leq\left(\int_{I_{n}}\left|u^{\prime}(t)\right|^{q} \mathrm{~d} t\right)^{\frac{p}{p+q}}\left(\int_{I_{n}} k^{p}(t) \mathrm{d} t\right)^{\frac{q}{p+q}},
$$


so from (43) we have:

$$
\begin{array}{r}
\alpha^{\frac{p q}{p+q}} \int_{I_{n}}\left|u^{\prime}(t)\right|^{\frac{q p^{2}}{p+q}} \mathrm{~d} t \leq C_{4}\left[\left(\int_{I_{n}}\left|u^{\prime}(t)\right|^{q} \mathrm{~d} t\right)^{\frac{p}{p+q}}\left(\int_{I_{n}} k^{p}(t) \mathrm{d} t\right)^{\frac{q}{p+q}}+\gamma^{\frac{p q}{p+q}}\right] \\
\leq C_{5}\left[\left(\int_{I_{n}}\left|u^{\prime}(t)\right|^{q} \mathrm{~d} t\right)^{\frac{p}{p+q}}+1\right] \leq C_{5}\left[\left(\int_{0}^{1}\left|u^{\prime}(t)\right|^{q} \mathrm{~d} t\right)^{\frac{p}{p+q}}+1\right]
\end{array}
$$

where $C_{5}$ is some constant independent with respect to $n$. From this inequality there exists some constant $C_{6}$ such that:

$$
\forall n \quad a_{n}=\int_{I_{n}}\left|u^{\prime}(t)\right|^{\frac{q p^{2}}{p+q}} \mathrm{~d} t \leq C_{6} .
$$

As $n \mapsto I_{n}$ is a nondecreasing sequence, the monotonous convergence theorem entails:

$$
\int_{0}^{1}\left|u^{\prime}(t)\right|^{\frac{q p^{2}}{p+q}} \mathrm{~d} t \leq C_{6}
$$

So we have proved that $u^{\prime}$ belongs to $L^{\frac{q p^{2}}{p+q}}(0,1)$.

Fourth case. If $k(t)=\left[\sum_{x \in \varphi^{-1}(t)} \frac{1}{\left|\varphi^{\prime}(x)\right|}\right]^{-1} \in L^{\delta}(0,1)$, with $q \leq \delta \leq 2<p<\infty$, then $u^{\prime} \in L^{\frac{\delta p q}{\delta+q}}(0,1)$.

As we have $q \leq \delta \leq 2<p<+\infty$, then thanks to the existence result of the problem (4), we have $u^{\prime} \in L^{q}(0,1)$. From (41) we obtain:

$$
\alpha^{\frac{\delta q}{\delta+q}} \int_{I_{n}}\left|u^{\prime}(t)\right|^{\frac{\delta p q}{\delta+q}} \mathrm{~d} t \leq C_{7}\left(\int_{I_{n}}\left|u^{\prime}(t)\right|^{\frac{\delta q}{\delta+q}} k^{\frac{\delta q}{\delta+q}}(t) \mathrm{d} t+\gamma^{\frac{\delta q}{\delta+q}}\right)
$$

Hölder's inequality shows that:

$$
\int_{I_{n}}\left|u^{\prime}(t)\right|^{\frac{\delta q}{\delta+q}} k^{\frac{\delta q}{\delta+q}}(t) \mathrm{d} t \leq\left(\int_{I_{n}}\left|u^{\prime}(t)\right|^{q} \mathrm{~d} t\right)^{\frac{\delta}{\delta+q}}\left(\int_{I_{n}} k^{\delta}(t) \mathrm{d} t\right)^{\frac{q}{\delta+q}}
$$

Thus from (44) we obtain:

$$
\begin{array}{r}
\alpha^{\frac{\delta q}{\delta+q}} \int_{I_{n}}\left|u^{\prime}(t)\right|^{\frac{\delta p q}{\delta+q}} \mathrm{~d} t \leq C_{7}\left[\left(\int_{I_{n}}\left|u^{\prime}(t)\right|^{q} \mathrm{~d} t\right)^{\frac{\delta}{\delta+q}}\left(\int_{I_{n}} k^{\delta}(t) \mathrm{d} t\right)^{\frac{q}{\delta+q}}+\gamma^{\frac{\delta q}{\delta+q}}\right] \\
\leq C_{8}\left[\left(\int_{I_{n}}\left|u^{\prime}(t)\right|^{q} \mathrm{~d} t\right)^{\frac{\delta}{\delta+q}}+1\right] \leq C_{8}\left[\left(\int_{0}^{1}\left|u^{\prime}(t)\right|^{q} \mathrm{~d} t\right)^{\frac{\delta}{\delta+q}}+1\right]
\end{array}
$$

where $C_{8}$ is some constant independent with respect to $n$. From this inequality there exists some constant $C_{9}$ such that:

$$
\forall n \quad a_{n}=\int_{I_{n}}\left|u^{\prime}(t)\right|^{\frac{\delta p q}{\delta+q}} \mathrm{~d} t \leq C_{9} .
$$

Since $n \mapsto I_{n}$ is a nondecreasing sequence, the monotonous convergence theorem gives:

$$
\int_{0}^{1}\left|u^{\prime}(t)\right|^{\frac{\delta p q}{\delta+q}} \mathrm{~d} t \leq C_{9}
$$


Thus we have proved that $u^{\prime} \in L^{\frac{\delta p q}{\delta+q}}(0,1)$; this means:

$$
\text { if } k(t)=\left[\sum_{x \in \varphi^{-1}(t)} \frac{1}{\left|\varphi^{\prime}(x)\right|}\right]^{-1} \in L^{\delta}(0,1) \text {, with } q \leq \delta \leq 2<p<\infty \text {, then } u^{\prime} \in L^{\frac{\delta p q}{\delta+q}}(0,1) .
$$

\section{Equivalent problem Without deviations}

Theorem 4.1. Let us assume hypotheses (i) to (iv) of Corollary 3.1 and

(v) $k(t)=\left[\sum_{x \in \varphi^{-1}(t)} \frac{1}{\left|\varphi^{\prime}(x)\right|}\right]^{-1} \in L^{p}(0,1)$, with $1 \leq q<p<\infty$;

(vi) for a.e. $x, \forall \xi, \eta \mapsto f(x, \eta, \xi)$ is strictly convex, and for a.e. $x, \forall \eta, \xi \mapsto f(x, \eta, \xi)$ is strictly convex.

Then every solution $u$ of the problem (4) belonging in $W_{0}^{1, q}(0,1)$ is solution of the following problem without deviations:

$$
\inf \left\{\int_{0}^{1} G\left(t, v(t), v^{\prime}\right)(t) \mathrm{d} t / v \in W_{0}^{1, p}(0,1)\right\}
$$

where $G(t, \eta, \xi)=\left\langle\nu_{t}^{\theta}, f\left(., \eta, u^{\prime} \circ \varphi().\right)\right\rangle+\left\langle\mu_{t}^{\varphi}, f(., u \circ \theta(),. \xi)\right\rangle$ for a.e. $t, \quad \forall \eta, \quad \forall \xi$.

Proof. From the assumption (v) and using Corollary 3.1, we have $u \in W_{0}^{1, p}(0,1)$. The function $(\eta, \xi) \longrightarrow$ $G(t, \eta, \xi)$ is strictly convex with the adequate growth. It follows that there exists a unique solution $w$ for the problem (45).

Moreover the problem (45) is equivalent to the resolution of its following Euler-Lagrange equation:

$$
\frac{\partial}{\partial \eta}\left\langle\nu_{t}^{\theta}, f\left(., w(t), u^{\prime} \circ \varphi(.)\right)\right\rangle-\frac{\mathrm{d}}{\mathrm{d} t}\left[\frac{\partial}{\partial \xi}\left\langle\mu_{t}^{\varphi}, f\left(., u \circ \theta(.), w^{\prime}(t)\right\rangle\right]=0 \text { in } \mathcal{D}_{t}^{\prime}(0,1) .\right.
$$

Let us recall that we can also rewrite the Euler-Lagrange equation of the problem (4) in the following form:

$$
\frac{\partial}{\partial \eta}\left\langle\nu_{t}^{\theta}, f\left(., u(t), u^{\prime} \circ \varphi(.)\right)\right\rangle-\frac{\mathrm{d}}{\mathrm{d} t}\left[\frac{\partial}{\partial \xi}\left\langle\mu_{t}^{\varphi}, f\left(., u \circ \theta(.), u^{\prime}(t)\right\rangle\right]=0 \text { in } \mathcal{D}_{t}^{\prime}(0,1),\right.
$$

where we use the notation:

$$
\frac{\partial}{\partial \eta}\left\langle\nu_{t}^{\theta}, f\left(., u(t), u^{\prime} \circ \varphi(.)\right)\right\rangle=\frac{\partial}{\partial \eta}\left\langle\nu_{t}^{\theta}, f\left(., \eta, u^{\prime} \circ \varphi(.)\right)\right\rangle_{\eta=u(t)}
$$

with the similar notation for the second term of equation (47).

From (47) $u$ is a solution of (46). Thus by the uniqueness of the solution of the problem (45) we obtain $u=w$.

\section{Necessary optimality Conditions of COntrol PRoblems Where the state PLAYS THE ROLE OF DEVIATION}

In this section, we establish necessary optimality conditions of the following control problem:

$$
\inf \left\{\int_{0}^{1} f_{1}\left(t, u(t), u^{\prime}(t), u \circ \theta_{v}(t), \theta_{v}(t), v(t)\right) \mathrm{d} t=J(u, v) /(u, v) \in E \times \mathcal{U}_{a d}\right\} .
$$

Under the state constraint, for instance,

$$
\left\{\begin{array}{l}
\frac{\mathrm{d} \theta_{v}(t)}{\mathrm{d} t}=g\left(t, \theta_{v}(t), v(t)\right) \\
\theta_{v}(0)=\theta_{0}, \quad 0<\theta_{0}<1, \quad \theta_{v}(t) \in[0,1] \quad \forall t
\end{array}\right.
$$


where $E$ stands for a subset of $W_{0}^{1, p}(0,1)$, for instance, and

$$
\mathcal{U}_{a d}=\left\{c \in L^{1}(0,1) / c(t) \geq 0, \int_{0}^{t} c(s) \mathrm{d} s \in[0,1] \quad \forall t \in[0,1]\right\} .
$$

To be clear we begin to consider this problem with a simple state equation in Section 5.1 and its application will be given in Section ??. Section 5.2 is devoted to the study of the general case.

\subsection{Problem where the constraint is simple}

The application of this section will be the financial model of $[9,10]$ given in Section ??. We consider in this section the following problem:

$$
\inf \left\{\int_{0}^{1} f_{1}\left(t, v(t), v^{\prime}(t), v \circ \theta_{c}(t), \theta_{c}(t), c(t)\right) \mathrm{d} t=J(v, c)\right\},
$$

where $(v, c)$ is a control, $\theta_{c}$ stands for the state of the system and satisfies the state constraint

$$
\left\{\begin{array}{l}
\frac{\mathrm{d} \theta_{c}(t)}{\mathrm{d} t}=c(t) \\
\theta_{c}(0)=0, \quad \theta_{c}(t) \in[0,1] \quad \forall t \in[0,1] .
\end{array}\right.
$$

The control $c$ belongs to

$$
\mathcal{U}_{a d}=\left\{c \in L^{1}(0,1) / c(t) \geq 0, \int_{0}^{t} c(s) \mathrm{d} s \in[0,1] \quad \forall t \in[0,1]\right\},
$$

and the control $v$ belongs to $W_{0}^{1, p}(0,1)$ with $p>1$. In the sequel to simplify, we denote $f_{1}$ by $f$. Let the following assumptions hold: $f:[0,1] \times \mathbb{R} \times \mathbb{R} \times \mathbb{R} \times[0,1] \times \mathbb{R}^{+} \longrightarrow \mathbb{R},\left(t, \eta_{1}, \xi, \eta_{2}, \nu, \lambda\right) \longrightarrow f\left(t, \eta_{1}, \xi, \eta_{2}, \nu, \lambda\right)$, continuously differentiable with respect to the five last arguments when $t$ is fixed, such that:

(i) $\left|f\left(t, \eta_{1}, \xi, \eta_{2}, \nu, \lambda\right)\right| \leq C_{1}\left(1+\left|\eta_{1}\right|^{p}+|\xi|^{p}+\left|\eta_{2}\right|^{p}+|\nu|^{p}+|\lambda|\right), C_{1}>0$.

(ii) There exists some positive constant $C_{2}$ such that

$$
\begin{aligned}
\left|\frac{\partial f}{\partial \eta_{1}}\left(t, \eta_{1}, \xi, \eta_{2}, \nu, \lambda\right)\right|+\left|\frac{\partial f}{\partial \eta_{2}}\left(t, \eta_{1}, \xi, \eta_{2}, \nu, \lambda\right)\right|+\left|\frac{\partial f}{\partial \nu}\left(t, \eta_{1}, \xi, \eta_{2}, \nu, \lambda\right)\right| & \\
+\left|\frac{\partial f}{\partial \xi}\left(t, \eta_{1}, \xi, \eta_{2}, \nu, \lambda\right)\right|+\left|\frac{\partial f}{\partial \lambda}\left(t, \eta_{1}, \xi, \eta_{2}, \nu, \lambda\right)\right| & \leq C_{2}\left(1+\left|\eta_{1}\right|^{p-1}+|\xi|^{p-1}+\left|\eta_{2}\right|^{p-1}+|\nu|^{p-1}\right), \\
& \text { for any } t, \eta_{1}, \eta_{2}, \nu, \xi, \lambda .
\end{aligned}
$$

There exists $(\bar{u}, \bar{c}) \in W_{0}^{1, p}(0,1) \times \mathcal{U}_{a d}$ solution of the problem (50)-(51)

$$
\text { such that } \bar{u}^{\prime} \circ \theta_{\bar{c}}(t) \text { exists } \forall t \in[0,1] \text {. }
$$

As the function $t \mapsto\left|\left\{s \in[0,1] / \bar{\theta}(s)=\theta_{\bar{c}}>t\right\}\right|$ is decreasing, we know that there exists a family of real numbers $\left(t_{i}\right)_{i \in I} \subset[0,1]$, with $I$ empty, finite or countable, such that:

$$
\left|\left\{s \in[0,1] / \bar{\theta}(s)=\theta_{\bar{c}}=t_{i}\right\}\right|>0 .
$$

Let also the following assumption hold: $\bar{\theta}=\theta_{\bar{c}}:[0,1] \longrightarrow[0,1]$ is absolutely continuous such that:

$$
\left\{\begin{array}{l}
\left|\bar{\theta}^{\prime}(.)\right| \in L^{\rho}(0,1), \frac{\mathcal{X}_{\bar{\theta}}(.)}{\left|\bar{\theta}^{\prime}(.)\right|} \in L^{\mu}(0,1), \operatorname{card}\left(\bar{\theta}^{-1}(.)\right) \in L^{\nu}(0,1) \\
\text { with } \frac{1}{\rho}+\frac{1}{p^{\prime}}+\frac{1}{\mu} \leq 1, \frac{1}{\mu}+\frac{1}{\nu}+\frac{1}{\rho}+\frac{1}{p^{\prime}}-\frac{1}{\nu}\left(\frac{1}{p^{\prime}}+\frac{1}{\mu}+\frac{1}{\rho}\right) \leq 1, \\
\frac{1}{p}+\frac{1}{p^{\prime}}=1
\end{array}\right.
$$


where

$$
\mathcal{X}_{\bar{\theta}}(.)=\mathcal{X}_{E}(.) \quad \text { with } E=\left\{s \in[0,1] / \bar{\theta}(s) \neq t_{i} \quad \forall i \in I\right\}
$$

Theorem 5.1. Under the assumptions (i), (ii), (52) and (54), the control $(\bar{u}, \bar{c})$ solution of the problem (50)-(51) satisfies the following relations:

(i) Equation

$$
\begin{aligned}
\frac{\partial f}{\partial \eta_{1}}(t, \bar{u}(t), \bar{c}(t))+ & \frac{\partial}{\partial \eta_{2}}\left\langle\mu_{t}, f\left(., \bar{u}(.), \bar{u}^{\prime}(.), \bar{u}(t), t, \bar{c}(.)\right)\right\rangle \\
& +\sum_{i \in I} \delta_{t_{i}} \int_{\left\{\bar{\theta}=t_{i}\right\}} \frac{\partial f}{\partial \eta_{2}}(s, \bar{u}(s), \bar{c}(s)) \mathrm{d} s-\frac{\mathrm{d}}{\mathrm{d} t}\left[\frac{\partial f}{\partial \xi}(t, \bar{u}(t), \bar{c}(t))\right]=0,
\end{aligned}
$$

in $\mathcal{D}_{t}{ }^{\prime}(0,1)$, where $f(s, \bar{u}(s), \bar{c}(s))=f\left(s, \bar{u}(s), \bar{u}^{\prime}(s), \bar{u} \circ \bar{\theta}(s), \bar{\theta}(s), \bar{c}(s)\right)$, and where $\mu_{t}$ stands for the counting measure defined by:

$$
\left\langle\mu_{t}, f\left(., \bar{u}(.), \bar{u}^{\prime}(.), \bar{u}(t), t,{ }_{,} c(.)\right)\right\rangle=\sum_{x \in \bar{\theta}_{\bar{c}}^{-1}(t)} \mathcal{X}_{\bar{\theta}}(x) \frac{f\left(x, \bar{u}(x), \bar{u}^{\prime}(x), \bar{u}(t), t, \bar{c}(x)\right)}{\left|\bar{\theta}^{\prime}(x)\right|}
$$

and $\delta_{t_{i}}$ is the Dirac measure at the point $t_{i}$.

(ii) Integrodifferential inequality

$$
\bar{c}(t)\left[\frac{\partial f}{\partial \lambda}(t, \bar{u}(t), \bar{c}(t))-\int_{t}^{1}\left(\frac{\partial f}{\partial \eta_{2}}(s, \bar{u}(s), \bar{c}(s)) \bar{u}^{\prime} \circ \bar{\theta}(s)+\frac{\partial f}{\partial \lambda}(s, \bar{u}(s), \bar{c}(s))\right) \mathrm{d} s\right] \leq 0
$$

for a.e. $t \in[0,1]$.

Proof. Let $(\bar{u}, \bar{c})$ be a solution of the problem (50)-(51), then we have:

$$
J(\bar{u}, \bar{c}) \leq J(v, c) \quad \forall(v, c) \in W_{0}^{1, p}(0,1) \times \mathcal{U}_{a d} .
$$

For any admissible $(v, c)$, for any $\epsilon>0,(\bar{u}+\epsilon v, \bar{c}+\epsilon(c-\bar{c}))$ is admissible and we obtain:

$$
\lim _{\epsilon \rightarrow 0} \frac{J(\bar{u}+\epsilon v, \bar{c}+\epsilon(c-\bar{c}))-J(\bar{u}, \bar{c})}{\epsilon} \geq 0 .
$$

By the regularity and the growth assumptions on $f$, using the Lebesgue theorem, we obtain the existence of the limit in (55), and thus we have the following inequality:

$$
\begin{array}{r}
\int_{0}^{1} \frac{\partial f}{\partial \eta_{1}}(t, \bar{u}(t), \bar{c}(t)) v(t) \mathrm{d} t+\int_{0}^{1} \frac{\partial f}{\partial \xi}(t, \bar{u}(t), \bar{c}(t)) v^{\prime}(t) \mathrm{d} t+\int_{0}^{1} \frac{\partial f}{\partial \eta_{2}}(t, \bar{u}(t), \bar{c}(t))\left(\bar{u}^{\prime} \circ \bar{\theta}(t) \delta(t)+v \circ \bar{\theta}(t)\right) \mathrm{d} t \\
+\int_{0}^{1} \frac{\partial f}{\partial \nu}(t, \bar{u}(t), \bar{c}(t)) \delta(t) \mathrm{d} t+\int_{0}^{1} \frac{\partial f}{\partial \lambda}(t, \bar{u}(t), \bar{c}(t))(c-\bar{c})(t) \mathrm{d} t \geq 0 \quad \forall(v, c) \in W_{0}^{1, p}(0,1) \times \mathcal{U}_{a d}
\end{array}
$$

where

$$
\bar{\theta}(t)=\int_{0}^{t} \bar{c}(s) \mathrm{d} s, \delta(t)=\int_{0}^{t}(c(s)-\bar{c}(s)) \mathrm{d} s .
$$


The inequality (56) is equivalent to (57) and (58):

$$
\begin{gathered}
\int_{0}^{1} \frac{\partial f}{\partial \eta_{1}}(t, \bar{u}(t), \bar{c}(t)) v(t) \mathrm{d} t+\int_{0}^{1} \frac{\partial f}{\partial \xi}(t, \bar{u}(t), \bar{c}(t)) v^{\prime}(t) \mathrm{d} t+\int_{0}^{1} \frac{\partial f}{\partial \eta_{2}}(t, \bar{u}(t), \bar{c}(t)) v \circ \bar{\theta}(t) \mathrm{d} t=0 \forall v \in W_{0}^{1, p}(0,1) \\
\int_{0}^{1} \frac{\partial f}{\partial \eta_{2}}(t, \bar{u}(t), \bar{c}(t)) \bar{u}^{\prime} \circ \bar{\theta}(t) \delta(t) \mathrm{d} t+\int_{0}^{1} \frac{\partial f}{\partial \nu}(t, \bar{u}(t), \bar{c}(t)) \delta(t) \mathrm{d} t+\int_{0}^{1} \frac{\partial f}{\partial \lambda}(t, \bar{u}(t), \bar{c}(t))(c-\bar{c})(t) \mathrm{d} t \geq 0 \forall c \in \mathcal{U}_{a d}
\end{gathered}
$$

Using the same idea as in the proof of Theorem 2.1, equation (57) is equivalent to:

$$
\begin{aligned}
\frac{\partial f}{\partial \eta_{1}}(t, \bar{u}(t), \bar{c}(t)) & +\frac{\partial}{\partial \eta_{2}}\left\langle\mu_{t}, f\left(., \bar{u}(.), \bar{u}^{\prime}(.), \bar{u}(t), t, \bar{c}(.)\right)\right\rangle \\
& +\sum_{i \in I} \delta_{t_{i}} \int_{\left\{\bar{\theta}=t_{i}\right\}} \frac{\partial f}{\partial \eta_{2}}(s, \bar{u}(s), \bar{c}(s)) \mathrm{d} s-\frac{\mathrm{d}}{\mathrm{d} t}\left[\frac{\partial f}{\partial \xi}(t, \bar{u}(t), \bar{c}(t))\right]=0 \text { in } \mathcal{D}_{t}^{\prime}(0,1) .
\end{aligned}
$$

This proves (i) of Theorem 5.1. By integrating by parts, (58) gives:

$$
\begin{array}{r}
\left.\int_{0}^{1} \frac{\partial f}{\partial \lambda}(t, \bar{u}(t), \bar{c}(t))(c-\bar{c})(t) \mathrm{d} t+\int_{0}^{1}\left[\int_{t}^{1}\left(\frac{\partial f}{\partial \eta_{2}}(s, \bar{u}(s), \bar{c}(s)) \bar{u}^{\prime} \circ \bar{\theta}(s)\right)+\frac{\partial f}{\partial \nu}(t, \bar{u}(t), \bar{c}(t))\right) \mathrm{d} s\right](c-\bar{c})(t) \mathrm{d} t \geq 0 \\
\forall c \in \mathcal{U}_{a d} \quad(59)
\end{array}
$$

Let $h \in \mathcal{D}(0,1)$ and $\gamma$ be such that: $-1 \leq h \leq 0$ and $0 \leq \gamma \leq 1$. Let us set $c=\bar{c}+\gamma h \bar{c}$ and let us show that $c$ is admissible:

$$
c=\bar{c}+\gamma h \bar{c}=\bar{c}(1+h \gamma) \geq \bar{c}(1-\gamma) \geq 0
$$

and

$$
0 \leq \int_{0}^{t} c(s) \mathrm{d} s=\int_{0}^{t} \bar{c}(s) \mathrm{d} s+\gamma \int_{0}^{t} h \bar{c}(s) \mathrm{d} s \leq \int_{0}^{t} \bar{c}(s) \mathrm{d} s \leq 1 .
$$

This proves that $c$ is admissible. Taking $c=\bar{c}+\gamma h \bar{c}$ in (59), we obtain:

$$
\begin{array}{r}
\left.\int_{0}^{1} \frac{\partial f}{\partial \lambda}(t, \bar{u}(t), \bar{c}(t)) \bar{c}(t) h(t) \mathrm{d} t+\int_{0}^{1}\left[\int_{t}^{1}\left(\frac{\partial f}{\partial \eta_{2}}(s, \bar{u}(s), \bar{c}(s)) \bar{u}^{\prime} \circ \bar{\theta}(s)\right)+\frac{\partial f}{\partial \nu}(t, \bar{u}(t), \bar{c}(t))\right) \mathrm{d} s\right] \bar{c}(t) h(t) \mathrm{d} t \geq 0 \\
\forall h \in \mathcal{D}(0,1), h \leq 0, \text { by homogeneousness }
\end{array}
$$

i.e.

$$
\frac{\partial f}{\partial \lambda}(t, \bar{u}(t), \bar{c}(t)) \bar{c}(t)+\left[\int_{t}^{1}\left(\frac{\partial f}{\partial \eta_{2}}(s, \bar{u}(s), \bar{c}(s)) \bar{u}^{\prime} \circ \bar{\theta}(s)\right)+\frac{\partial f}{\partial \nu}(t, \bar{u}(t), \bar{c}(t))\right] \bar{c}(t) \leq 0 \quad \text { a.e. } t \in[0,1] .
$$

This proves (ii) of Theorem 5.1.

\subsection{Problem where the constraint is general}

We consider in this section the following problem:

$$
\inf \left\{\int_{0}^{1} f\left(t, u(t), u^{\prime}(t), u \circ \theta_{v}(t), \theta_{v}(t), v(t)\right) \mathrm{d} t=J(u, v)\right\}
$$

under the state constraint,

$$
\left\{\begin{array}{l}
\frac{\mathrm{d} \theta_{v}(t)}{\mathrm{d} t}=g(t, \theta(t), v(t)) \\
\theta_{v}(0)=\theta_{0}, \quad 0<\theta_{0}<1, \quad \theta_{v}(t) \in[0,1] \quad \forall t
\end{array}\right.
$$


where the control $v$ belongs to $L^{\infty}((0,1), \mathcal{V})$ for instance, where $\mathcal{V}=[0,1]$ and the control $u$ belongs to $W_{0}^{1, p}(0,1)$ with $p>1$. Let the following assumption hold:

$f:[0,1] \times \mathbb{R} \times \mathbb{R} \times \mathbb{R} \times[0,1] \times \mathbb{R} \longrightarrow \mathbb{R},\left(t, \eta_{1}, \xi, \eta_{2}, \nu, \lambda\right) \longrightarrow f\left(t, \eta_{1}, \xi, \eta_{2}, \nu, \lambda\right)$, continuously differentiable in the five last arguments when $t$ is fixed. We assume that $f$ satisfies the hypotheses (i) and (ii) of Section 5.1 and the fonction $g$ satisfies the following assumptions:

$g:[0,1] \times[0,1] \times \mathbb{R} \longrightarrow \mathbb{R},(t, \alpha, \beta) \longrightarrow g(t, \alpha, \beta)$, continuously differentiable with respect to $\alpha$, twice continuously differentiable with respect to $\beta$ and continuous with respect to $t$. We assume that there exists a constant $K$ such that:

$$
\left|\frac{\partial g}{\partial \alpha}(t, \alpha, \beta)\right|+\left|\frac{\partial g}{\partial \beta}(t, \alpha, \beta)\right| \leq K .
$$

Let us suppose that:

$$
\begin{aligned}
& \exists(\bar{u}, \bar{v}) \in W_{0}^{1, p}(0,1) \times L^{p}((0,1), \mathcal{V}) \text { solution of the problem }(61)-(62) \\
& \text { such that } \bar{u}^{\prime} \circ \theta_{\bar{v}}(t) \text { exists } \forall t \in[0,1] .
\end{aligned}
$$

As the function $t \mapsto\left|\left\{s \in[0,1] / \bar{\theta}(s)=\theta_{\bar{v}}>t\right\}\right|$ is decreasing, it is known that there exists a family of real members $\left(t_{i}\right)_{i \in I} \subset[0,1]$ with $I$ empty, finite or countable, such that:

$$
\left|\left\{s \in[0,1] / \bar{\theta}(s)=\theta_{\bar{v}}=t_{i}\right\}\right|>0 .
$$

Let also the following assumption hold:

$\left.\bar{\theta}=\theta_{\bar{v}}:[0,1] \longrightarrow\right] 0,1[$ is absolutely continuous such that:

$$
\left\{\begin{array}{l}
\left|\bar{\theta}^{\prime}(.)\right| \in L^{\rho}(0,1), \frac{\mathcal{X}_{\bar{\theta}}(.)}{\left|\theta^{\prime}(.)\right|} \in L^{\mu}(0,1), \operatorname{card}\left(\bar{\theta}^{-1}(.)\right) \in L^{\nu}(0,1) \\
\text { with } \frac{1}{\rho}+\frac{1}{p^{\prime}}+\frac{1}{\mu} \leq 1, \frac{1}{\mu}+\frac{1}{\nu}+\frac{1}{\rho}+\frac{1}{p^{\prime}}-\frac{1}{\nu}\left(\frac{1}{p^{\prime}}+\frac{1}{\mu}+\frac{1}{\rho}\right) \leq 1 \\
\frac{1}{p}+\frac{1}{p^{\prime}}=1
\end{array}\right.
$$

where

$$
\mathcal{X}_{\bar{\theta}}(.)=\mathcal{X}_{E}(.) \text { with } E=\left\{s \in[0,1] / \bar{\theta}(s) \neq t_{i} \quad \forall i \in I\right\} .
$$

Theorem 5.2. Under the assumptions (64) and (66), the control $(\bar{u}, \bar{v})$ solution of the problem (61)-(62) satisfies the following relations:

(i) $\frac{\partial f}{\partial \eta_{1}}(t, \bar{u}(t), \bar{v}(t))+\frac{\partial}{\partial \eta_{2}}\left\langle\mu_{t}, f\left(., \bar{u}(),. \bar{u}^{\prime}(),. \bar{u}(t), t, \bar{v}().\right)\right\rangle+\sum_{i \in I} \delta_{t_{i}} \int_{\left\{\bar{\theta}=t_{i}\right\}} \frac{\partial f}{\partial \eta_{2}}(s, \bar{u}(s), \bar{v}(s)) \mathrm{d} s$ $-\frac{\mathrm{d}}{\mathrm{d} t}\left[\frac{\partial f}{\partial \xi}(t, \bar{u}(t), \bar{v}(t))\right]=0$,

in $\mathcal{D}_{t}^{\prime}(0,1)$, with the notation $f(s, \bar{u}(s), \bar{c}(s))=f\left(s, \bar{u}(s), \bar{u}^{\prime}(s), \bar{u} \circ \bar{\theta}(s), \bar{\theta}(s), \bar{v}(s)\right)$. In addition $\mu_{t}$ stands for the counting measure defined by:

$$
\left\langle\mu_{t}, f\left(., \bar{u}(.), \bar{u}^{\prime}(.), \bar{u}(t), t, \bar{v}(.)\right)\right\rangle=\sum_{x \in \bar{\theta}^{-1}(t)} \mathcal{X}_{\bar{\theta}}(x) \frac{f\left(x, \bar{u}(x), \bar{u}^{\prime}(x), \bar{u}(t), t, \bar{v}(x)\right)}{\left|\bar{\theta}^{\prime}(x)\right|},
$$

and $\delta_{t_{i}}$ is the Dirac measure at the point $t_{i}$.

(ii) $\bar{v}(t)\left[\frac{\partial f}{\partial \lambda}(t, \bar{u}(t), \bar{v}(t))+\bar{p}(t) \frac{\partial g}{\partial \beta}(t, \bar{\theta}(t), \bar{v}(t))\right] \leq 0$ for a.e. $t \in[0,1]$, 
where $\bar{p}(t)$ is the adjoint state solution of the equation:

$$
\left\{\begin{array}{l}
\bar{p}^{\prime}(t)=-\frac{\partial g}{\partial \alpha}(t, \bar{\theta}(t), \bar{v}(t)) \bar{p}(t)-\left[\frac{\partial f}{\partial \eta_{2}}(t, \bar{u}(t), \bar{v}(t)) \bar{u}^{\prime} \circ \bar{\theta}(t)\right. \\
\left.+\frac{\partial f}{\partial \nu}(t, \bar{u}(t), \bar{v}(t))\right] \text { on }[0,1] \\
\bar{p}(1)=0 .
\end{array}\right.
$$

Proof. Assume that there exists $(\bar{u}, \bar{v})$ solution of the problem (61)-(62) i.e.

$$
J(\bar{u}, \bar{v}) \leq J(u, v) \quad \forall(u, v) \in W_{0}^{1, p}(0,1) \times L^{\infty}((0,1), \mathcal{V}) .
$$

We have

$$
J(\bar{u}+\epsilon u, \bar{v}+\epsilon(v-\bar{v}))-J(\bar{u}, \bar{v}) \geq 0 \quad \forall \epsilon>0 \text { small enough, } \quad \forall(u, v) \text { admissible. }
$$

Let us set $\bar{\theta}$ the state optimal trajectory i.e. the solution of (62) corresponding to $\bar{v}$. Moreover, if we set $\theta_{\epsilon}$ the solution of the state equation (62) corresponding to $v_{\epsilon}=\bar{v}+\epsilon(v-\bar{v})$, then we have: $\frac{\mathrm{d} \theta_{\epsilon}}{\mathrm{d} t}(t)=g\left(t, \theta_{\epsilon}(t), v_{\epsilon}(t)\right)$ and $\frac{\mathrm{d} \bar{\theta}(t)}{\mathrm{d} t}=g(t, \bar{\theta}(t), \bar{v}(t))$. Following [14] let us consider $x($.$) the solution of the following trajectory equation:$

$$
\left\{\begin{array}{l}
x^{\prime}(t)=\frac{\partial g}{\partial \alpha}(t, \bar{\theta}(t), \bar{v}(t)) x(t)+\frac{\partial g}{\partial \beta}(t, \bar{\theta}(t), \bar{v}(t))(v-\bar{v})(t) \text { on }(0,1) \\
x(0)=0
\end{array}\right.
$$

and let us set, for any $\epsilon>0$ small enough,

$$
y_{\epsilon}(.)=\frac{\theta_{\epsilon}(.)-\bar{\theta}(.)}{\epsilon}-x(.) .
$$

Thus we have:

$$
\begin{aligned}
y_{\epsilon}(t)= & \int_{0}^{t} \frac{1}{\epsilon}\left[g\left(s, \theta_{\epsilon}(s), v_{\epsilon}(s)\right)-g(s, \bar{\theta}(s), \bar{v}(s))\right] \mathrm{d} s \\
& -\int_{0}^{t}\left[\frac{\partial g}{\partial \alpha}(s, \bar{\theta}(s), \bar{v}(s)) x(s)+\frac{\partial g}{\partial \beta}(s, \bar{\theta}(s), \bar{v}(s))(v-\bar{v})(s)\right] \mathrm{d} s .
\end{aligned}
$$

By using the mean value theorem, the Gronwall lemma and the assumption (63), we obtain $\left\|y_{\epsilon}\right\|_{\infty} \longrightarrow 0$ when $\epsilon \longrightarrow 0$. Therefore we have:

$$
\theta_{\epsilon}(t)=\bar{\theta}(t)+\epsilon x(t)+o(\epsilon) \quad \forall t \in[0,1],
$$

and using the Taylor-Lagrange formula, we obtain:

$$
\begin{aligned}
J(\bar{u}+\epsilon u, \bar{v}+\epsilon(v-\bar{v}))= & J(\bar{u}, \bar{v})+\epsilon\left[\int_{0}^{1} \frac{\partial f}{\partial \eta_{1}}(t, \bar{u}(t), \bar{v}(t)) u(t) \mathrm{d} t\right. \\
& +\int_{0}^{1} \frac{\partial f}{\partial \xi}(t, \bar{u}(t), \bar{v}(t)) u^{\prime}(t) \mathrm{d} t+\int_{0}^{1} \frac{\partial f}{\partial \eta_{2}}(t, \bar{u}(t), \bar{v}(t))\left[\bar{u}^{\prime} \circ \bar{\theta}(t) x(t)+u \circ \bar{\theta}(t)\right] \mathrm{d} t \\
& \left.+\int_{0}^{1} \frac{\partial f}{\partial \nu}(t, \bar{u}(t), \bar{v}(t)) x(t) \mathrm{d} t+\int_{0}^{1} \frac{\partial f}{\partial \lambda}(t, \bar{u}(t), \bar{v}(t))(v-\bar{v})(t) \mathrm{d} t\right]+o(\epsilon) .
\end{aligned}
$$


From (67), we obtain

$$
\begin{aligned}
& \int_{0}^{1} \frac{\partial f}{\partial \eta_{1}}(t, \bar{u}(t), \bar{v}(t)) u(t) \mathrm{d} t+\int_{0}^{1} \frac{\partial f}{\partial \xi}(t, \bar{u}(t), \bar{v}(t)) u^{\prime}(t) \mathrm{d} t+\int_{0}^{1} \frac{\partial f}{\partial \eta_{2}}(t, \bar{u}(t), \bar{v}(t))\left[\bar{u}^{\prime} \circ \bar{\theta}(t) x(t)+u \circ \bar{\theta}(t)\right] \mathrm{d} t \\
& \quad+\int_{0}^{1} \frac{\partial f}{\partial \nu}(t, \bar{u}(t), \bar{v}(t)) x(t) \mathrm{d} t+\int_{0}^{1} \frac{\partial f}{\partial \lambda}(t, \bar{u}(t), \bar{v}(t))(v-\bar{v})(t) \mathrm{d} t \geq 0 \quad \forall u \in W_{0}^{1, p}(0,1), \quad \forall v \in L^{p}((0,1), \mathcal{V}) .
\end{aligned}
$$

Following [14] let us introduce the adjoint state $\bar{p}(t)$ solution of the following equation

$$
\left\{\begin{aligned}
\bar{p}^{\prime}(t) & =-\frac{\partial g}{\partial \alpha}(t, \bar{\theta}(t), \bar{v}(t)) \bar{p}(t)-\left[\frac{\partial f}{\partial \eta_{2}}(t, \bar{u}(t), \bar{v}(t)) \bar{u}^{\prime} \circ \bar{\theta}(t)\right. \\
& \left.+\frac{\partial f}{\partial \nu}(t, \bar{u}(t), \bar{v}(t))\right] \text { on }[0,1] \\
\bar{p}(1) & =0 .
\end{aligned}\right.
$$

Let us consider the following integral which follows from (69)

$$
\int_{0}^{1}\left[\frac{\partial f}{\partial \eta_{2}}(t, \bar{u}(t), \bar{v}(t)) \bar{u}^{\prime} \circ \bar{\theta}(t)+\frac{\partial f}{\partial \nu}(t, \bar{u}(t), \bar{v}(t))\right] x(t) \mathrm{d} t .
$$

Using (70), we rewrite (71) in the following form:

$$
\int_{0}^{1}\left[\frac{\partial f}{\partial \eta_{2}}(t, \bar{u}(t), \bar{v}(t)) \bar{u}^{\prime} \circ \bar{\theta}(t)+\frac{\partial f}{\partial \nu}(t, \bar{u}(t), \bar{v}(t))\right] x(t) \mathrm{d} t=-\int_{0}^{1}\left[\bar{p}^{\prime}(t)+\frac{\partial g}{\partial \alpha}(t, \bar{\theta}(t), \bar{v}(t)) \bar{p}(t)\right] x(t) \mathrm{d} t .
$$

By integrating by parts the right hand side of (72), using (68) we obtain:

$$
\int_{0}^{1}\left[\frac{\partial f}{\partial \eta_{2}}(t, \bar{u}(t), \bar{v}(t)) \bar{u}^{\prime} \circ \bar{\theta}(t)+\frac{\partial f}{\partial \nu}(t, \bar{u}(t), \bar{v}(t))\right] x(t) \mathrm{d} t=\int_{0}^{1} \bar{p}(t) \frac{\partial g}{\partial \beta}(t, \bar{\theta}(t), \bar{v}(t))(v-\bar{v})(t) \mathrm{d} t .
$$

Finally we rewrite (69) in the following form:

$$
\begin{aligned}
& \int_{0}^{1} \frac{\partial f}{\partial \eta_{1}}(t, \bar{u}(t), \bar{v}(t)) u(t) \mathrm{d} t+\int_{0}^{1} \frac{\partial f}{\partial \xi}(t, \bar{u}(t), \bar{v}(t)) u^{\prime}(t) \mathrm{d} t+\int_{0}^{1} \frac{\partial f}{\partial \eta_{2}}(t, \bar{u}(t), \bar{v}(t)) u \circ \bar{\theta}(t) \mathrm{d} t \\
& +\int_{0}^{1}\left[\frac{\partial f}{\partial \lambda}(t, \bar{u}(t), \bar{v}(t))+\bar{p}(t) \frac{\partial g}{\partial \beta}(t, \bar{\theta}(t), \bar{v}(t))\right](v-\bar{v})(t) \mathrm{d} t \geq 0 \quad \forall u \in W_{0}^{1, p}(0,1), \quad \forall v \in L^{p}((0,1), \mathcal{V}) .
\end{aligned}
$$

The inequality (73) is equivalent to the following inequalities (74) and (75):

$$
\begin{gathered}
\int_{0}^{1} \frac{\partial f}{\partial \eta_{1}}(t, \bar{u}(t), \bar{v}(t)) u(t) \mathrm{d} t+\int_{0}^{1} \frac{\partial f}{\partial \xi}(t, \bar{u}(t), \bar{v}(t)) u^{\prime}(t) \mathrm{d} t+\int_{0}^{1} \frac{\partial f}{\partial \eta_{2}}(t, \bar{u}(t), \bar{v}(t)) u \circ \bar{\theta}(t) \mathrm{d} t=0 \\
\forall u \in W_{0}^{1, p}(0,1),
\end{gathered}
$$

and

$$
\int_{0}^{1}\left[\frac{\partial f}{\partial \lambda}(t, \bar{u}(t), \bar{v}(t))+\bar{p}(t) \frac{\partial g}{\partial \beta}(t, \bar{\theta}(t), \bar{v}(t))\right](v-\bar{v})(t) \mathrm{d} t \geq 0 \quad \forall v \in L^{p}((0,1), \mathcal{V}) .
$$


Using the same idea as in the proof of Theorem 2.1 we show that the inequality (74) is equivalent to:

$$
\begin{aligned}
& \frac{\partial f}{\partial \eta_{1}}(t, \bar{u}(t), \bar{c}(t))+\frac{\partial}{\partial \eta_{2}}\left\langle\mu_{t}, f\left(., \bar{u}(.), \bar{u}^{\prime}(.), \bar{u}(t), t, \bar{c}(.)\right)\right\rangle \\
& \quad+\sum_{i \in I} \delta_{t_{i}} \int_{\left\{\bar{\theta}=t_{i}\right\}} \frac{\partial f}{\partial \eta_{2}}(s, \bar{u}(s), \bar{c}(s)) \mathrm{d} s-\frac{\mathrm{d}}{\mathrm{d} t}\left[\frac{\partial f}{\partial \xi}(t, \bar{u}(t), \bar{c}(t))\right]=0,
\end{aligned}
$$

in $\mathcal{D}_{t}{ }^{\prime}(0,1)$, with the short notation $f(s, \bar{u}(s), \bar{c}(s))=f\left(s, \bar{u}(s), \bar{u}^{\prime}(s), \bar{u} \circ \theta(s), \theta(s), \bar{v}(s)\right)$. Let $h$ and $\gamma$ be such that $h \in \mathcal{D}(0,1),-1 \leq h \leq 0$ and $0<\gamma<1$ and let us set $v=\bar{v}+\gamma h \bar{v}$. By taking $v=\bar{v}+\gamma h \bar{v}$ in (75) we obtain:

$$
\int_{0}^{1}\left[\frac{\partial f}{\partial \lambda}(t, \bar{u}(t), \bar{v}(t))+\bar{p}(t) \frac{\partial g}{\partial \beta}(t, \bar{\theta}(t), \bar{v}(t))\right] \bar{v}(t) h(t) \mathrm{d} t \geq 0 \quad \forall h \in \mathcal{D}(0,1), h \leq 0, \text { by homogeneousness. }
$$

This gives:

$$
\bar{v}(t)\left[\frac{\partial f}{\partial \lambda}(t, \bar{u}(t), \bar{v}(t))+\bar{p}(t) \frac{\partial g}{\partial \beta}(t, \bar{\theta}(t), \bar{v}(t))\right] \leq 0 \text { a.e. } t \in[0,1]
$$

\section{Application to a financial market problem: the model of Jouini, Koenl And Touzi $[9,10]$}

We shall consider the regular case as in [9]. Following [9] let us present this model. The financial market consists in one riskless asset, whose price function is given by $S(t)$. We assume that sales are subject to taxes on benefits following the usual first-in-first-out rule according to which any bond sold at some time $t$ should be the oldest one in the time $t$ portofolio. We introduce the set $\triangle=\left\{(t, u) \in \mathbb{R}^{2} / 0 \leq u \leq t \leq 1\right\}$. For each monetary unit invested at time $u$ and sold out at time $t$, we denote by $\varphi(t, u)$ the after tax amount received at time $t ; \varphi(t, u)$ is assumed to be $c^{1}$ decreasing in $t$ and increasing in $u$. Let $x(t)$ be the investment rate in units of the risky asset at time $t$; the disinvestment rate of this asset at time $t$ is $y(t)=x \circ \theta(t) \theta^{\prime}(t)$ for a.e. $t$ (cf. [9]) where $\theta($.$) is the delay function given by:$

$$
\theta(t)=\sup \left[s \in[0,1] / \int_{0}^{s} x(u) \mathrm{d} u \leq \int_{0}^{t} y(u) \mathrm{d} u\right] .
$$

We have: $\theta(0)=0,0 \leq \theta(t) \leq t$. The trading strategy is such that $\int_{0}^{t} y(s) \mathrm{d} s \leq \int_{0}^{t} x(s) \mathrm{d} s$. The following two functions are useful for the formulation of the problem: $z(t)=x(t) . S(t)$ and $v(t) \geq 0$, such that $\theta^{\prime}(t)=v(t)$. With these functions, the agent control problem consists in maximizing the utility functional:

$$
J(z, v)=\int_{0}^{1} U\left(t, c^{z, \theta_{v}}(t)\right) \mathrm{d} t
$$

under the state constraint:

$$
\frac{\mathrm{d} \theta_{v}}{\mathrm{~d} t}=v, \quad \theta_{v}(0)=0 \text { with } v(t) \geq 0,
$$

and the control $(z, v)$ belongs to $\mathcal{U}_{a d}=\left\{(z, v) / 0 \leq z(t) \leq \omega(t), v(t) \geq 0, \int_{0}^{t} v(s) \mathrm{d} s \leq t\right\}$. The agent consumption rate $c^{z, \theta_{v}}$ is defined by

$$
c^{z, \theta_{v}}(t)=\omega-z(t)+\theta^{\prime}(t) z(\theta(t)) \cdot \varphi(t, \theta(t)) .
$$

In [10], the authors show that this problem has an optimal solution $(\bar{x}, \bar{v})$ such that $\bar{x}$ is piecewise $c^{1}$ on $[0,1]$ and $\bar{v}$ is piecewise continuous on $[0,1]$. Let $\bar{\theta}$ be the optimal state, corresponding to $(\bar{x}, \bar{v})$. In this paper, 
we assume a regularity assumption on the optimal solution. As the function $t \mapsto\left|\left\{s \in[0,1] / \bar{\theta}(s)=\theta_{\bar{v}}>t\right\}\right|$ is decreasing, there exists a family of reals $\left(t_{i}\right)_{i \in I} \subset[0,1]$, with $I$ empty, finite or countable such that:

$$
\left|\left\{s \in[0,1] / \bar{\theta}(s)=\theta_{\bar{v}}=t_{i}\right\}\right|>0 .
$$

Let the following assumption hold: $\bar{\theta}=\theta_{\bar{v}}:[0,1] \longrightarrow[0,1]$ is absolutely continuous such that:

$$
\left\{\begin{array}{l}
\left|\bar{\theta}^{\prime}(.)\right| \in L^{\rho}(0,1), \frac{\mathcal{X}_{\bar{\theta}}(.)}{\left|\theta^{\prime}(.)\right|} \in L^{\mu}(0,1), \operatorname{card}\left(\bar{\theta}^{-1}(.)\right) \in L^{\nu^{\prime}}(\mathbb{R}) \\
\text { with } \frac{1}{\rho}+\frac{1}{p^{\prime}}+\frac{1}{\mu} \leq 1, \frac{1}{\mu}+\frac{1}{\nu}+\frac{1}{\rho}+\frac{1}{p^{\prime}}-\frac{1}{\nu}\left(\frac{1}{p^{\prime}}+\frac{1}{\mu}+\frac{1}{\rho}\right) \leq 1, \\
\frac{1}{p}+\frac{1}{p^{\prime}}=1,
\end{array}\right.
$$

where

$$
\mathcal{X}_{\bar{\theta}}(.)=\mathcal{X}_{E}(.) \text { with } E=\left\{s \in[0,1] / \bar{\theta}(s) \neq t_{i} \quad \forall i \in I\right\} .
$$

Under regularity assumption on $(\bar{x}, \bar{v})$ and under the following assumptions:

$U:[0,1] \times \mathbb{R}^{+} \longrightarrow \mathbb{R},(t, \xi) \longrightarrow U(t, \xi)$ is continuously differentiable in $\xi$ when $t$ is fixed such that:

(i) $|U(t, \xi)| \leq C_{7}\left(1+|\xi|^{p}\right), C_{7}>0$

(ii) $\left|\frac{\partial U}{\partial \xi}(t, \xi)\right| \leq C_{8}\left(1+|\xi|^{p-1}\right), C_{8}>0$.

Our previous results allow us to obtain the following:

\section{Corollary 6.1.}

(i) $\bar{x}(t)\left[-\frac{\partial U}{\partial \xi}(t, \bar{c}(t))+\left\langle\nu_{t}^{\bar{\theta}}, \frac{\partial U}{\partial \xi}(., \bar{c}(.) \varphi(., t) \bar{v}()).\right\rangle\right]+\bar{x}(t) \sum_{i \in I} \delta_{t_{i}} \int_{\left\{\bar{\theta}=t_{i}\right\}} \frac{\partial U}{\partial \xi}(s, \bar{c}(s)) \varphi(s, \bar{\theta}(s)) \mathrm{d} s \geq 0$ in $\mathcal{D}_{t}{ }^{\prime}(0,1)$, where we denote $\bar{\theta}=\theta_{\bar{v}}, \bar{c}(t)=c(\bar{x}, \bar{v})(t)=\omega(t)-\bar{x}(t)+\bar{v}(t) \cdot \bar{x} \circ \theta_{\bar{v}}(t) \cdot \varphi\left(t, \theta_{\bar{v}}(t)\right)$. The measure $\nu_{t}^{\bar{\theta}}$ is the counting measure defined by:

$$
\left\langle\nu_{t}^{\bar{\theta}}, \frac{\partial U}{\partial \xi}(., \bar{c}(.) \varphi(., t) \bar{v}(.))\right\rangle=\sum_{x \in \bar{\theta}^{-1}(t)} \mathcal{X}_{\bar{\theta}}(x) \frac{\frac{\partial U}{\partial \xi}(x, \bar{c}(x) \varphi(x, t) \bar{v}(x))}{\left|\bar{\theta}^{\prime}(x)\right|} \text { for a.e.t. }
$$

(ii) $\bar{v}(t)\left[\frac{\partial U}{\partial \xi}(t, \bar{c}(t)) \cdot \bar{x} \circ \bar{\theta}(t) \cdot \varphi(t, \bar{\theta}(t))\right]+\bar{v}(t)\left\{\int_{t}^{1} \bar{v}(s) \cdot \frac{\partial U}{\partial \xi} \cdot\left[\bar{x}^{\prime} \circ \bar{\theta}(s) \cdot \varphi(s, \bar{\theta}(s))+\bar{x} \circ \bar{\theta}(s) \frac{\partial \varphi}{\partial \theta}(s, \bar{\theta}(s))\right] \mathrm{d} s\right\}$ $\geq 0$ for a.e. $t \in[0,1]$.

Remark 6.1. In [9] $\bar{\theta}$ is supposed to be implicitly invertible. This is not the case here by (77). Let us point out that the conditions above are some what local, contrary to which exists in the literature, if we except the case of translations [13] and the case of strictly monotonous deviations $[9,12]$.

Proof of Corollary ??. Let $(\bar{x}, \bar{v})$ be an optimal solution of the agent problem which belongs to the set of admissibles controls:

$$
\mathcal{U}_{a d}=\left\{(x, v) / 0 \leq x(t) \leq \omega(t), v(t) \geq 0, \int_{0}^{t} v(s) \mathrm{d} s \leq t\right\},
$$

and let us assume that $\bar{x}$ is piecewise $c^{1}$ and $\bar{v}$ is piecewise continuous on $[0,1]$. Then we have:

$$
J(\bar{x}, \bar{v}) \geq J(x, v) \quad \forall(x, v) \in \mathcal{U}_{a d} .
$$


For all $(y, r)$ admissible, for any $\epsilon>0$, the control $(\bar{x}+\epsilon(y-\bar{x}), \bar{v}+\epsilon(r-\bar{v}))$ is admissible and we obtain:

$$
\lim _{\epsilon \rightarrow 0} \frac{J(\bar{x}+\epsilon(y-\bar{x}), \bar{v}+\epsilon(r-\bar{v}))-J(\bar{x}, \bar{v})}{\epsilon} \leq 0 .
$$

By the regularity and the growth assumptions on $U$, we obtain the existence of the limit in (79) and thus we have the following inequality:

$$
\begin{aligned}
\int_{0}^{1} \frac{\partial U}{\partial \xi}(t, \bar{c}(t))[-(y(t)-\bar{x}(t)) & +(y-\bar{x}) \circ \bar{\theta}(t) \cdot \bar{v}(t) \cdot \varphi(t, \bar{\theta}(t))] \mathrm{d} t \\
& +\int_{0}^{1} \frac{\partial U}{\partial \xi}(t, \bar{c}(t)) \bar{x} \circ \bar{\theta}(t) \varphi(t, \bar{\theta}(t))(r(t)-v(t)) \mathrm{d} t \\
+ & \int_{0}^{1} \frac{\partial U}{\partial \xi}(t, \bar{c}(t))\left[\bar{v}(t) \bar{x}^{\prime} \circ \bar{\theta}(t) \varphi(t, \bar{\theta}(t))+\bar{v}(t) \bar{x} \circ \bar{\theta}(t) \frac{\partial \varphi}{\partial \theta}(t, \bar{\theta}(t))\right] \lambda(t) \mathrm{d} t \leq 0
\end{aligned}
$$

for any admissible $(y, r)$, where $\lambda$ is the increase in $\bar{\theta}$ corresponding to the increase $r$ in the control i.e. $\lambda^{\prime}=r-\bar{v}$, $\lambda(0)=0$. By integrating by parts we obtain:

$$
\begin{aligned}
\int_{0}^{1} \frac{\partial U}{\partial \xi}(t, \bar{c}(t))[ & \left.\bar{v}(t) \bar{x}^{\prime} \circ \bar{\theta}(t) \varphi(t, \bar{\theta}(t))+\bar{v}(t) \bar{x} \circ \bar{\theta}(t) \frac{\partial \varphi}{\partial \theta}(t, \bar{\theta}(t))\right] \lambda(t) \mathrm{d} t \\
=[- & \left.\int_{t}^{1} \frac{\partial U}{\partial \xi}(s, \bar{c}(s))\left[\bar{v}(s) \bar{x}^{\prime} \circ \bar{\theta}(s) \varphi(s, \bar{\theta}(s))+\bar{v}(s) \bar{x} \circ \bar{\theta}(s) \frac{\partial \varphi}{\partial \theta}(s, \bar{\theta}(s))\right] \mathrm{d} s \lambda(t)\right]_{0}^{1} \\
& \quad+\int_{0}^{1}\left\{\int_{t}^{1} \frac{\partial U}{\partial \xi}(s, \bar{c}(s))\left[\bar{v}(s) \bar{x}^{\prime} \circ \bar{\theta}(s) \varphi(s, \bar{\theta}(s))+\bar{v}(s) \bar{x} \circ \bar{\theta}(s) \frac{\partial \varphi}{\partial \theta}(s, \bar{\theta}(s))\right] \mathrm{d} s\right\}(r-\bar{v})(t) \mathrm{d} t .
\end{aligned}
$$

We rewrite the inequality (80) in the following form:

$$
\begin{aligned}
\int_{0}^{1}\left[\frac{\partial U}{\partial \xi}(t, \bar{c}(t))\right. & \bar{v}(t) \varphi(t, \bar{\theta}(t))(y-\bar{x}) \circ \bar{\theta}(t)] \mathrm{d} t \\
& +\int_{0}^{1} \frac{\partial U}{\partial \xi}(t, \bar{c}(t)) \bar{x} \circ \bar{\theta}(t) \varphi(t, \bar{\theta}(t))(r-\bar{v})(t) \mathrm{d} t+\int_{0}^{1}\left[-\frac{\partial U}{\partial \xi}(t, \bar{c}(t))(y(t)-\bar{x}(t))\right] \mathrm{d} t \\
& +\int_{0}^{1}\left\{\int_{t}^{1} \frac{\partial U}{\partial \xi}(s, \bar{c}(s))\left[\bar{v}(s) \bar{x}^{\prime} \circ \bar{\theta}(s) \varphi(s, \bar{\theta}(s))+\bar{v}(s) \bar{x} \circ \bar{\theta}(s) \frac{\partial \varphi}{\partial \theta}(s, \bar{\theta}(s))\right] \mathrm{d} s\right\}(r-\bar{v})(t) \mathrm{d} t \leq 0
\end{aligned}
$$

for any admissible $(y, r)$. We can also easily derive from (81) the following inequality

$$
\begin{aligned}
\int_{0}^{1} \frac{\partial U}{\partial \xi}(t, \bar{c}(t)) \bar{x} \circ \bar{\theta}(t) \varphi(t, \bar{\theta}(t))(r-\bar{v})(t) \mathrm{d} t & \\
& +\int_{0}^{1}\left\{\int_{t}^{1} \frac{\partial U}{\partial \xi}(s, \bar{c}(s))\left[\bar{v}(s) \bar{x}^{\prime} \circ \bar{\theta}(s) \varphi(s, \theta(s))+\bar{v}(s) \bar{x} \circ \bar{\theta}(s) \frac{\partial \varphi}{\partial \theta}(s, \bar{\theta}(s))\right] \mathrm{d} s\right\}(r-\bar{v})(t) \mathrm{d} t \leq 0
\end{aligned}
$$

for any admissible $r$, and

$$
\int_{0}^{1}\left[-\frac{\partial U}{\partial \xi}(t, \bar{c}(t))(y(t)-\bar{x}(t))\right] \mathrm{d} t+\int_{0}^{1}\left[\frac{\partial U}{\partial \xi}(t, \bar{c}(t)) \bar{v}(t) \varphi(t, \bar{\theta}(t))(y-\bar{x}) \circ \bar{\theta}(t)\right] \mathrm{d} t \leq 0
$$


for any admissible $y$. And as in the proof of Theorem 2.1, we can rewrite the inequality (83) in the following form:

$$
\begin{aligned}
\int_{0}^{1}\left[-\frac{\partial U}{\partial \xi}(t, \bar{c}(t))+\left\langle\nu_{t}^{\bar{\theta}}, \frac{\partial U}{\partial \xi}(., \bar{c}(.)) \varphi(., t) \bar{v}(.)\right\rangle\right] & (y(t)-\bar{x}(t)) \mathrm{d} t \\
& +\left\langle\sum_{i \in I} \delta_{t_{i}} \int_{\left\{\bar{\theta}=t_{i}\right\}} \frac{\partial U}{\partial \xi}(s, \bar{c}(s)) \varphi(s, \bar{\theta}(s)) \mathrm{d} s, y-\bar{x}\right\rangle \leq 0, \text { for any admissible } y
\end{aligned}
$$

Let $h$ and $\gamma$ be such that $h \in \mathcal{D}(0,1),-1 \leq h \leq 0$ and $0<\gamma<1$. Let us set $y=\bar{x}+\gamma h \bar{x}$ and let us show that $y$ is admissible. We have:

and

$$
y(t)=x(t)(1+\gamma h(t)) \geq \bar{x}(t)(1-\gamma) \geq 0
$$

$$
\omega(t)-y(t)=\omega(t)-\bar{x}(t)-\gamma h \bar{x}(t) \geq \omega(t)-\bar{x}(t) \geq 0
$$

i.e. $y$ is admissible. If we take in (84) $y=\bar{x}+\gamma h \bar{x}$, then we obtain:

$$
\begin{aligned}
\int_{0}^{1}\left[-\frac{\partial U}{\partial \xi}(t, \bar{c}(t))+\left\langle\nu_{t}^{\theta}, \frac{\partial U}{\partial \xi}(., \bar{c}(.)) \varphi(., t) \bar{v}(.)\right\rangle\right] \bar{x}(t) h(t) \mathrm{d} t & \\
& +\left\langle\bar{x}(t) \sum_{i \in I} \delta_{t_{i}} \int_{\left\{\bar{\theta}=t_{i}\right\}} \frac{\partial U}{\partial \xi}(s, \bar{c}(s)) \varphi(s, \bar{\theta}(s)) \mathrm{d} s, h\right\rangle \leq 0
\end{aligned}
$$

for any $h \in \mathcal{D}(0,1), h \leq 0$, by homogeneousness. We can therefore rewrite the inequality (85) in the following form:$$
\bar{x}(t)\left[-\frac{\partial U}{\partial \xi}(t, \bar{c}(t))+\left\langle\nu_{t}^{\bar{\theta}}, \frac{\partial U}{\partial \xi}(., \bar{c}(.)) \varphi(., t) \bar{v}(.)\right\rangle\right]+\bar{x}(t) \sum_{i \in I} \delta_{t_{i}} \int_{\left\{\bar{\theta}=t_{i}\right\}} \frac{\partial U}{\partial \xi}(s, \bar{c}(s)) \varphi(s, \bar{\theta}(s)) \mathrm{d} s \geq 0, \text { in } \mathcal{D}^{\prime}(0,1) .
$$

This proves (i) of Corollary ??. We can also rewrite the inequality (82) in the form:

$$
\begin{array}{r}
\int_{0}^{1}\left[\frac{\partial U}{\partial \xi}(t, \bar{c}(t)) \bar{x} \circ \bar{\theta}(t) \varphi(t, \bar{\theta}(t))\right](r-\bar{v})(t) \mathrm{d} t+\int_{0}^{1}\left[\int_{t}^{1} \frac{\partial U}{\partial \xi}(s, \bar{c}(s)) \bar{v}(s) \bar{x}^{\prime} \circ \bar{\theta}(s) \varphi(s, \bar{\theta}(s)) \mathrm{d} s\right](r-\bar{v})(t) \mathrm{d} t \\
+\int_{0}^{1}\left[\int_{t}^{1}\left[\bar{v}(s) \bar{x} \circ \bar{\theta}(s) \frac{\partial \varphi}{\partial \theta}(s, \bar{\theta}(s))\right] \mathrm{d} s\right](r-\bar{v})(t) \mathrm{d} t \leq 0
\end{array}
$$

for any admissible $r$. Let us consider the following control $r(t)=\bar{v}(t)+\bar{v}(t) \gamma h(t)$. This control is admissible since we have:

and

$$
r(t)=\bar{v}(t)(1+\gamma h(t)) \bar{v}(t)(1-\gamma) \geq 0
$$

$$
\int_{0}^{t} r(s) \mathrm{d} s=\int_{0}^{t} \bar{v}(s) \mathrm{d} s+\gamma \int_{0}^{t} \bar{v}(s) h(s) \mathrm{d} s \leq \int_{0}^{t} \bar{v}(s) \mathrm{d} s \leq t
$$

If we take in the inequality $(87), r(t)=\bar{v}(t)+\bar{v}(t) \gamma h(t)$, then we obtain:

$$
\begin{aligned}
\int_{0}^{1}\left[\frac{\partial U}{\partial \xi}(t, \bar{c}(t)) \bar{x} \circ \bar{\theta}(t) \varphi(t, \bar{\theta}(t))\right] \bar{v}(t) h(t) \mathrm{d} t+\int_{0}^{1} & {\left[\int_{t}^{1} \frac{\partial U}{\partial \xi}(s, \bar{c}(s)) \bar{v}(s) \bar{x}^{\prime} \circ \bar{\theta}(s) \varphi(s, \bar{\theta}(s)) \mathrm{d} s\right] \bar{v}(t) h(t) \mathrm{d} t } \\
& +\int_{0}^{1}\left[\int_{t}^{1}\left[\bar{v}(s) \bar{x} \circ \bar{\theta}(s) \frac{\partial \varphi}{\partial \theta}(s, \bar{\theta}(s))\right] \mathrm{d} s\right] \bar{v}(t) h(t) \mathrm{d} t \leq 0
\end{aligned}
$$


for any $h \in \mathcal{D}(0,1), h \leq 0$, by homogeneousness, i.e.

$$
\begin{aligned}
\bar{v}(t)\left[\frac{\partial U}{\partial \xi}(t, \bar{c}(t)) \bar{x} \circ \bar{\theta}(t) \varphi(t, \bar{\theta}(t))\right]+\bar{v}(t)\left[\int_{t}^{1} \frac{\partial U}{\partial \xi}(s, \bar{c}(s)) \bar{v}(s) \bar{x}^{\prime} \circ \bar{\theta}(s) \varphi(s, \bar{\theta}(s)) \mathrm{d} s\right] & \\
& +\bar{v}(t)\left[\int_{t}^{1}\left[\bar{v}(s) \bar{x} \circ \bar{\theta}(s) \frac{\partial \varphi}{\partial \theta}(s, \bar{\theta}(s))\right] \mathrm{d} s\right] \geq 0 \text { in } \mathcal{D}^{\prime}(0,1)
\end{aligned}
$$

this proves (ii) of Corollary ??.

Acknowledgements. The authors thank A. Chambolle for his help to prove Proposition 2.1.

\section{REFERENCES}

[1] G. Carlier and R. Tahraoui, On some optimal control problems governed by a state equation with memory. ESAIM: COCV (to appear).

[2] M. Drakhlin, On the variational problem in the space of absolutely continuous functions. Nonlin. Anal. TMA 23 (1994) $1345-1351$.

[3] M. Drakhlin and E. Litsyn, On the variation problem for a family of functionals in the space of absolutly continuous functions. Nonlin. Anal. TMA 26 (1996) 463-468.

[4] M.E. Drakhlin and E. Stepanov, On weak lower semi-continuity for a class of functionals with deviating argument. Nonlin. Anal. TMA 28 (1997) 2005-2015.

[5] M.E. Drakhlin, E. Litsyn and E. Stepanov, Variational methods for a class of nonlocal functionals. Comput. Math. Appl. 37 (1999) 79-100.

[6] L.C. Evans and R.F. Gariepy, Measure theory and fine properties of functions. CRC Press, Inc. (1992).

[7] L. Freddi, Limits of control problems with weakly converging nonlocal input operators. Calculus of variations and optimal control (Haifa, 1998), Math. 411, Chapman Hall/CRC, Boca Raton, FL (2000) 117-140.

[8] A.A. Gruzdev and S.A. Gusarenko, On reduction of variational problems to extremal problems without constraints. Russians mathematics 38 (1994) 37-47.

[9] E. Jouini, P.F. Koehl and N. Touzi, Optimal investment with taxes: an optimal control problem with endogeneous delay. Nonlin. Anal. TMA 37 (1999) 31-56.

[10] E. Jouini, P.F. Koehl and N.Touzi, Optimal investment with taxes: an existence result. J. Math. Economics 33 (2000) 373-388.

[11] G.A. Kamenskii, Variational and boundary value problems with deviating argument. Diff. Equ. 6 (1970) 1349-1358.

[12] G.A. Kamenskii, On some necessary conditions of functionals with deviating argument. Nonlin. Anal. TMA 17 (1991) $457-464$.

[13] G.A. Kamenskii, Boundary value problems for differential-difference equations arising from variational problems. Nonlin. Anal. TMA 18 (1992) 801-813.

[14] P.L. Lions and B. Larrouturou, Optimisation et commande optimale, méthodes mathématiques pour l'ingénieur, cours de l'École Polytechnique, Palaiseau, France.

[15] L. Samassi, Calculus of variation for funtionals with deviating arguments. Ph.D. thesis, University Paris-Dauphine, France (2004).

[16] L. Samassi and R. Tahraoui, Comment établir des conditions nécessaires d'optimalité dans les problèmes de contrôle dont certains arguments sont déviés ? C.R. Acad. Sci. Paris Ser. 338 (2004) 611-616.

[17] J.A. Wheeler and R.P. Feynman, Classical electrodynamics in term of direct interparticle actions. Rev. Modern Phys. 21 (1949) 425-433. 